\title{
Environmental Monitoring WSN
}

\author{
Ittipong Khemapech \\ University of the Thai Chamber of Commerce, \\ Thailand
}

\section{Introduction}

Energy conservation is currently growing in importance. This chapter focuses on the issue of energy conservation within the domain of Wireless Sensor Network (WSN). There are also specific reasons why energy conservation is more important for WSN than for other types of networks. A WSN consists of multiple sensors which are able to sense some aspect of their environment and communicate their readings to a base station or sink without being physically connected to it. Sensors are often also resource constrained, being small in size and relying on small batteries for power. Consequently, the efficient utilisation of energy should be an important priority for designing WSN network protocols. This differs from the traditional approach to designing network protocols where issues like survivability, maximising throughput or reliability have been prioritised. Making energy conservation an important design priority is a new approach.

Wireless sensor network (WSN) is an important research area with a major technological impact. With significant breakthroughs in "Micro Electromechanical Systems", or MEMS, (Warneke \& Pister, 2002), sensors are becoming smaller. It is feasible to fit them into a smaller volume with more power and less production costs. WSN may be deployed in a wide range of different environments. These include remote and hostile environments as well as local and friendly ones. A major driving force behind research in WSN has been military and surveillance applications. Recently, however diversification has occurred with the development of civil applications. One example which is used as a reference point throughout this work is Great Duck Island (GDI). Sensors were scattered over a remote island to monitor the seabird's migration (Mainwaring et al., 2002). In another example WSN was deployed around volcanoes (Allen et al., 2006). Such applications illustrate the usefulness of WSN which make data collection feasible from remote and hostile environments with minimal human intervention.

One of the main objectives of WSN power conservation is to minimise energy usage whilst other functional requirements such as reliability or time synchronisation are still achieved. Some authors argue that multi hop communication allows for deployment in scenarios where direct communication with a base station is not practical (Arora et al., 2004; Allen et al., 2006; Chintalapudi et al., 2006). However, the spread of the Internet means that wireless devices may often communicate directly with a device that is connected to the Internet and has a reliable power supply. This work focuses on the design of wireless sensor networks protocols where direct communication with a powered base station is feasible and data is sent from the sensors to the base station at regular intervals. There are several important scenarios where such two assumptions hold. 
This research work specifically looks at WSN where direct communication is possible and beneficial. A protocol for WSN, Power \& Reliability Aware Protocol (PoRAP), is developed and provides energy efficient data delivery, without increasing packet loss. In designing PoRAP several experiments were conducted to establish the relationship between transmission power, reception signal strength and packet reception success. These showed a strong correlation between Received Signal Strength Indicator (RSSI) and Packet Reception Rate (PRR). In PoRAP, the RSSI is monitored at the base station. If the RSSI is too high the base station signals the sensor to reduce its transmission level, thereby saving power. If the RSSI is too low the base station signals the sensor to increase its transmission level so that packet loss is avoided.

PoRAP adopts a schedule based scheme for the sources' transmissions. It is assumed that nodes will be reporting measurement data regularly back to the base station. Each reporting interval consists of three time periods. In the first the base station sends a configuration packet. This informs nodes whether they are to increase, decrease or leave unaltered their transmission levels. There are then slots, each of which contains a data slot within which a sensor may transmit its data to the base station. There may then be a period of quiet before the start of a new cycle. Delays and clock drifts are measured so that nodes know when to wake up to listen and transmit. Delays depend upon payload size.

The design aims to optimise energy conservation rather than system throughput, in many sensing scenarios high throughput is not required. Sensors collect and report some physical data such as temperature and humidity. In such cases, the data reporting rate may be in minutes or hours. Two packet structures are used in PoRAP. The control packet is used in control and setup phase. It contains essential information for transmission power adaptation and scheduling. The data packet is used to deliver the collected physical data back to the base station.

The remaining parts of this chapter is organised as follows: Section 2 addressed application specific WSN. At present, WSN has been used in both military and civil applications. Each application category has particular characteristics and its own set of requirements. Hence, there are significant challenges in a generic protocol design for a variety of applications. Resource constraint issues are provided in Section 3. Apart from limited power resources, sensors also have constrained communication ranges for indoor and outdoor environments. The distance between the source and destination is crucial to employing an appropriate underlying communication paradigm. Section 4 describes the experimental details and their results which motivate the design of PoRAP. There are several factors which affect the link quality metrics such as distance between source and base station and time of day. The design of PoRAP is outlined in Section 5. PoRAP consists of several TinyOS components at the source and base station. The results shown in Section 4 motivates the design. The results of PoRAP evaluation in terms of energy conservation are presented in Section 6. Lower transmission power can be used to save the power whilst the reliability is in the desired range. Finally, Section 7 concludes the chapter.

\section{Application specific WSN}

Apart from being used in military or surveillance, WSN has been deployed in several civil applications which have different requirements. Periodic sensing is required in some habitat and environmental monitoring systems whilst event sensing is the norm in surveillance systems. Network lifetime and data reporting rates are therefore major concerns for the 
former and latter cases, respectively. To be application specific results in a more complicated design process, especially in the case of designing a generic power-aware protocol.

In total, seven groups of applications have been categorised by us based upon their functionalities including habitat monitoring (HM) (Juang et al., 2002; Mainwaring et al., 2002; Szewczyk et al., 2004), environmental monitoring (EM) (Allen et al., 2006; Martinez et al., 2005), health monitoring (HEM) (Jovanov et al., 2003, Otto et al., 2006), structural health monitoring (SHM) (Chintalapudi et al., 2006; Kottapalli et al., 2003; Paek et al., 2005, Schmid et al., 2005), event detection and tracking (EDT) (Arora et al., 2004; Dreicer et al., 2002; Simon et al., 2004), transport monitoring (TM) (Coleri et al., 2004) and location-aware system (LAS) (Brignone et al., 2005). Specific capabilities and underlying communication paradigms have been outlined. For example, data encryption may be required in some health monitoring systems for transmitting a patient's diagnosis data to the main server located at the hospital. Furthermore, data correctness is also required in this case. In some applications such as event tracking and detection systems, several intermediate nodes are required for forwarding the sensed data to the base station. However, a direct communication from source to base station is found in some health monitoring systems. This section addresses application specific characteristics of WSN applications by detailing the differences in their requirements.

\subsection{Event/periodic based}

The "Event/Periodic Based" aspect demonstrates how often data reporting is conducted. There are three main types including event-based, periodic-based and hybrid. Each sensor is triggered to operate by the occurrence of an event in the case of an event-based application. An example of this application type is the Event Detection and Tracking. Congestion is one of the major concerns designing a protocol to support event-based networking as it is caused by a lot of traffic generated by all sources in an event area. The key idea of congestion avoidance is to control data reporting rate of such sensors (Sankarasubramaniam et al., 2003). However, the main assumption is that all data packets have the same priority. Packet loss is therefore tolerantly acceptable. There are several works on congestion control specifically developed for WSN (Ee \& Bajcsy, 2004; Hull et al., 2004, Lu et al., 2002, Wan et al., 2003). The congestion control approach focuses on channel monitoring to dynamically adjust the data forwarding rate. CODA (Wan et al., 2003) has been designed to cover two types of problems corresponding to the deployed sensors and their data rate. However, it does not provide any queue occupancy monitoring. Sending an ACK (Acknowledgement) in the case of persistent congestion, even if it is small in size, may increase the number of traffic. This mechanism also requires feedback signalling which results in higher cost. Only packet prioritisation could be found in (Lu et al., 2002). However, it proposes the VMS (Velocity Monotonic Scheduling) policy which supports both static and dynamic computation of the requested velocity and it also solves the fairness problem. Both channel and queue occupancy monitoring are provided in (Hull et al., 2004) and (Ee \& Bajcsy, 2004). A child node can transmit packets only when its parent does not experience congestion problems and some help from the MAC (Medium Access Control) layer to shift the transmitting time to avoid interference are proposed in (Hull et al., 2004). A similar concept also exists in (Ee \& Bajcsy, 2004) by comparing the normalised rate of a node and its parents.

Each sensor periodically performs its operation. Some examples of data collected by the sensors are temperature and humidity. The significant change in readings may be used to 
identify the presence of seabirds (Mainwaring et al., 2002) and intruders (Arora et al., 2004). Instead of heavily generated traffics, both sensor and network lifetimes are the core requirement of this application type. Finally, both event and periodic sensing operations may be desired in some applications such as SHM (Structural Health Monitoring) and EDT systems. For example, the displacement of construction elements is periodically reported for maintenance purposes whilst an event-based operation is applied for warning and evacuating notifications during an earthquake.

This work focuses on developing a power-aware protocol which supports an efficient data delivery in periodic based applications such as health, habitat and environmental monitoring where the data reporting rate is in minutes or hours. Sensors may be scattered over a remote and hostile area to collect and report physical data and they should have to operate for months. Hence, battery lifetime is important and one of the main goals is to conserve communication energy.

\subsection{Mobility of sources}

The mobility of sources or sensors can be found in some particular applications such as HM (Habitat Monitoring, HEM (HEalth Monitoring and LAS (Location-Aware System). In some cases, sensors are attached to the targeted objects or location (Jovanov et al., 2003; Juang et al., 2002, Martinez et al., 2005) in order to monitor the data of interest or current location. Mobile sensor networks have a different set of supporting infrastructures compared to the traditional WSN. It is essential for each mobile sensor to know its own location. The GPS (Global Positioning System) is used for locating sensors which are attached to the goods. Alternatively, several nodes with known locations may be used as references for the others to calculate their own locations [Brignone et al., 2005]. The issues of sensor mobility are beyond the scope of this work.

\subsection{Mobility of sources}

Wireless sensor network (WSN) consists of sensors which are wirelessly connected. The main objective of WSN development is to collect physical data from an area of interest. Therefore, communication between sensors is a key aspect. Normally there are two node types in WSN including the source and base station. Sources are ordinary sensors having limited resources whereas base stations are assumed to have more power and other resources. The main duty of sensors is collecting and transmitting data to the destination or base station. The sensors are probably required to cover a large area and direct communication between sources and base station is unlikely due to limited communication range. Several intermediate sensors responsible for forwarding data packets to the base station are therefore required. This is known as multi-hop communication. Each sensor also acts as a routing node in order to find the shortest or cheapest path by means of power consumption. Several applications deploy multi-hop communication (Allen et al., 2006; Chintalapudi et al., 2006; Schmid et al., 2005; Dreicer et al., 2002; Simon et al., 2004). The multi-hop approach has several advantages. For example, a new path is discovered when some sensors die. Deploying a large number of cheap sensors over a large area is feasible as the sensors can act as routing nodes and the collected data is forwarded to the destination. However, one of its drawbacks is each node has to listen to the channel most of the time in order to detect if a message is arriving. The sensors have to conduct some computations in order to discover the cheapest path. Moreover, communication with its neighbours is another requirement to set up a selected path. Such processes require a significant amount 
of power, taken from the battery power available. Introducing several intelligent features to each sensor is also limited due to the power constraint.

Each source can transmit the data directly to the base station if the sources are located within the base station's communication range. Some examples of existing applications deploying single-hop communication (Mainwaring et al., 2002; Martinez et al., 2005; Jovanov et al., 2003; Otto et al., 2006). For single-hop, the sources are located within the base station's range. Direct communication is therefore feasible and several benefits are realised. One of the advantages is the ability to introduce a variety of intelligent features to the base station as it is assumed to have more power and computational capabilities compared to an ordinary sensor. Each source does not require the power necessary for routing. Idle listening can be minimised as the sources can be switched to sleep mode if they do not transmit data or receive the control packet. The base station controls the communication schedule of its sources to avoid data collisions. Power for carrier sensing is not desired. In multi-hop, each source is responsible for sensing, data reporting and routing. The number of transmissions and receptions increases with the number of intermediary nodes required for data forwarding.

This work looks at protocol development for single-hop. A scenario where the single-hop is viable is Environmental Monitoring (EM). Sources and base stations are distributed and several clusters or patches are formed. A power-aware, single-hop protocol can thus be used in each of the clusters (Mainwaring et al., 2002). A low duty cycle is the norm in EM so the communication cycle of each source can be scheduled by the base station. A time slot is allocated to each source to perform data transmissions. Carrier sensing is thus not required in this scheme. The sources synchronise to the base station by checking the information included in the control packet.

\subsection{Reliability}

Wireless sensor network (WSN) has been currently deployed in several civil applications. The physical data is collected and transmitted for further analysis. The issue of reliability in data delivery is important for providing complete reliability consumes a significant proportion of power. Applying the Transmission Control Protocol (TCP) protocol to WSN is expensive because of its three-way handshake mechanism and packet header size. The User Datagram Protocol (UDP) is considered to be more suitable for sensors although it was designed to provide unreliable data transport. In some applications, data loss may be not a serious problem because of the large amount of deployed sensors. Reliable data transport is important for some types of data such as control messages delivered by the base station (Wan et al., 2002). The following paragraphs provide some details of reliable transport protocol for WSN researches including PSFQ (Pump Slowly, Fetch Quickly) (Wan et al., 2002), ESRT (Event-to-Sink Reliable Transport) (Sankarasubramaniam et al., 2003), and RMST (Reliable Multi-Segment Transport) (Stann \& Heidemann, 2003).

One of the main goals to achieve reliable data transport is to orchestrate data receiving and forwarding processes to lessen the packet loss due to buffer overflow. PSFQ proposes three different operations including pump, fetch and report. Data generated from a source node is injected slowly into the network in order to allow such nodes experiencing data loss to fetch the missing packets very aggressively. Timing is a core process in order to avoid operational synchronisation. A hop-by-hop recovery is applied to avoid exponential error accumulation as occurs in the end-to-end scheme. Data delivery status information can be sent back to users or applications in a piggyback fashion. 
Focusing only on the forward or sensor-to-sink direction, ESRT was designed to provide a reliable data transport by inspecting current network state in terms of reliability and congestion. The state result is categorised and the reporting frequency is then repetitively adjusted to reach an optimal point. ESRT provides both reliable data transport and congestion control. Local buffer level monitoring is used to detect congestion.

Directed Diffusion (Intanagonwiwat et al., 2003) is a routing protocol which provides a multipoint-to-multipoint communication. A sink firstly indicates an interest and propagates it to the nodes. Interest and node information is kept as gradients. The optimised reinforced path is then established to send the attribute-value pairs data. RMST is implemented as a filter to provide some information about the data fragment such as ID and total number of fragments to detect loss. A NACK (Negative ACKnowledgement) is sent via a back-channel to upstream neighbouring nodes in case of data loss.

According to the above fundamental protocol descriptions, several conclusions can be made. In a densely deployed environment, data loss may be accepted. However, this condition may apply only in the case of sensor-to-sink traffic. The sink or base station plays a major role in the network by broadcasting several control packets to the sensors. Such packets should not be lost. Moreover, there are various types of sensing data, such as structural displacement due to wind or earthquake (Xu et al., 2004), which need some combination from different nodes to create usable data before forwarding that data to the sink. PSFQ designing concepts are more complicated but can be applied to a broader area of application. The data retransmission mechanisms are not mentioned in ESRT as it focuses on statistical reliability. However, PSFQ does not provide congestion control schemes as ESRT does. RMST is designed to run over the Directed Diffusion routing protocol. Although it may take the least effort compared to the other two, it is not generic enough.

\section{Resource constraint issues}

This section introduces several issues of resource constraint in WSN. A sensor can be considered as a small computing device which is capable of sensing, data processing, storage and communication. Sensors are deployed in an area of interest and they may have to operate without maintenance throughout their lifetimes. Power is thus one of the limited resources. Unless an external source of energy is provided, power for all operations comes from batteries. Two AA batteries are required in the widely used platforms such as Tmote, Telos and Mica. The capacity of the AA battery is approximately 2,000 to 3,000 milli-amperehour (mAh). In order to calculate the battery life, the capacity is divided by the actual load current and the obtained lifetime is in hours. An equation for calculating sensor's lifetime is given in (Polastre et al., 2004) where the lifetime is equal to the product between capacity $(\mathrm{mAh})$ and voltage $(3 \mathrm{~V})$ divided by total energy consumption in micro-joules. The default capacity defined in (Polastre et al., 2004) is set at 2,500mAh.

Communication accounts for a significant proportion of energy consumption. There are four main modes of communication including sending, receiving, sleeping and listening. The transceiver is one of the major sensor components and it makes them capable of communicating with other nodes. Recent transceivers or radio chips such as CC1000 and CC2420 provide programmable transmission power. Sensors consume less power when they send at a lower power level. Hence, transmission power control is one of power-aware schemes in WSN. The sensors do not always send at the maximum power. Tmote platform is chosen in this study and it employs CC2420 transceiver. For the CC2420 mote the 
minimum and maximum transmission power is 8.5 and 17.4 milli-amperes (mA). Over $50 \%$ of the power can be saved if the minimum power is always used.

Sensors equipped with CC2420 radio chips consume a greater amount of power when they receive data. According to the data sheet, $19.7 \mathrm{~mA}$ is required for reception. Listening and sleeping consume 365 and 20 micro-amperes $(\mu \mathrm{A})$, respectively. Hence, in the case of the CC2420 mote, data reception consumes more energy than transmission. The base station is the destination and it may be connected to a desktop or laptop computer. In such cases, the base station has extra power from the connected machine. However, the sensors which act as intermediary nodes between source and destination have to receive and forward packets resulting in sensor's lifetimes being decreased. The listening power is approximately 17 times greater than sleeping. In some applications such as environmental monitoring, the data sampling interval may be in minutes or hours. The transceivers should be switched to sleep mode instead of listening. Scheduling issues occur when two nodes communicate with each other. The data is not received if the receiver is in sleep mode. The nodes have to agree upon the same scheduling. Another scheme based upon contention-based can be used; the receiver can periodically listen to the signal propagated over the medium to inspect whether the incoming message is destined for it.

WSN is also a shared medium system. Each of the sources and base station has to engage the medium to perform data communication. Data collisions occur if the sources transmit at the same time and energy will be wasted by unsuccessful data delivery. A Medium Access Control (MAC) protocol is required to resolve the contention. The features of the MAC protocol together with the application behaviour determine when a node is idle, when it is listening and when it is sending. As each of these states have different power requirements the MAC protocol impacts upon the efficiency of operation and the power consumption. There are two main MAC schemes; the contention and the schedule based. The medium is sensed prior to transmission and the sensors have to backoff if the medium is declared busy. This work focuses on the single-hop where the sources send data directly to the base station. Another scheme, schedule based, is adopted. A data slot is allocated to each node. No carrier sensing and corresponding energy is required. The main issue is that the slot must be long enough for completing data delivery, otherwise, data collisions are likely. Experimentations required to determine the duration required for both sending and receiving together with the effective factors such as data payload size. Each node is switched to sleep mode to spend the least amount of power when its slot does not arrive.

The buffering capacity of CC2420 is limited to 128 bytes. Taking the header's and footer's sizes into account, the allowable data payload size is thus less than 128 bytes. Apart from sensed data, some control information is required in the packet such as identification and timestamp. Additional packet structures may be required if all the information cannot be stored in one packet. Control overhead is considered as one of the costs and should be minimised in order to decrease transmission and reception energy.

Wireless sensor network (WSN) has been currently deployed in several surveillance and civil applications. Sensors may be scattered over an area of interest which can be very large. The communication range is thus important and depends upon the selected transceiver. For example, the CC2420 mote has $50 \mathrm{~m}$ and $125 \mathrm{~m}$ indoor and outdoor ranges. Under some circumstances, the maximum transmission power may not produce the maximum ranges. Furthermore, sending data to the node located at farther distances requires higher transmission power. Multi-hop is therefore usually used in WSN. Several intermediary sensors are required for data forwarding from the source to destination. Single-hop 
communication is feasible if the destination is located within the source's range. Multiple transmissions and receptions are not required if direct communication applies. However, the same transmission power cannot always be used as the link quality changes over time. The next section describes several sources of variability in radio frequency

\section{Motivation of PoRAP development}

This work aims at building a communication protocol for WSN. The targeted scenario is the periodic-based where a low duty cycle is required. The network consists of a fixed set of sources and a base station. Furthermore, direct data communications between the base station and its sources are feasible. The communication protocol to be developed will effectively support the single-hop WSN. Such a structure forms a network cluster which can be used in some environmental or habitat monitoring system such as (Mainwaring et al., 2002) and (Tolle et al., 2005). As the number of sources is fixed throughout the communications, the data reporting rate is fairly constant. The communication of the sources can be therefore scheduled and controlled by the base station. A time slot is allocated to each source and will be used for data communication. Only one source can use the shared medium whilst the others switch to sleep mode by turning their radios off and consuming the least amount of energy. Data collision can be avoided and idle listening can be minimised.

\subsection{Sensor node power consumption}

This section establishes the significance of network communication as a consumer of energy within a wireless sensor network. In doing so a careful reading of sensor data sheets is used to inform calculations based upon the sensor's parameters and simulations. What proportion of the power is used for communication is investigated and how power may be conserved is identified.

In order to investigate how power is consumed by a sensor, a simulation study has been established. The results are validated by the CC1000 transceiver data sheet. As the sensor operating system used in this work is TinyOS, the selected simulator is TOSSIM which is a TinyOS library. TinyOS is an operating system specifically designed for embedded devices such as sensors. It has been widely used in both research and commercial communities. The selected release of the simulator is TOSSIM 1 and it does not provide power usage measurement capability. PowerTOSSIM, an extension module developed for analysing power consumption of hardware components (Shnayder et al., 2004) is used to address the investigation on power consumption and it is included in Tiny 1.1.11. The only sensor platform supported in PowerTOSSIM is Mica2 which employed the CC1000 radio chip. The PowerTOSSIM supports an operating frequency of 400 Megahertz (MHz) and a voltage of 3 Volt. The energy model file of PowerTOSSIM adopts the required transmission current for each power level. According to the CC1000 datasheet, 31 output power levels ranging from 20 to $+10 \mathrm{dBm}$ can be programmed. The $\mathrm{dBm}$ is the measurement of power loss in decibels $(\mathrm{dB})$ using 1 milli-watt $(\mathrm{mW})$ as a reference value.

\subsubsection{Simulation parameters}

A sensor node was created in the simulation and performs as a transmitting node. An experiment was conducted to obtain the current consumption required by each transmission power level. In total five transmission powers including $-20,-10,0,+6$ and $+10 \mathrm{dBm}$ were 
used. The corresponding current consumption was measured by (Shnayder et al., 2004) and their results are shown in Table 1. A simulation duration of 60 seconds and a total of 30 runs were conducted at each power level. A higher current will be consumed if the sensor transmits at a higher power.

\begin{tabular}{|c|c|}
\hline Transmission Power $\mathbf{( d B m )}$ & Required Current $\mathbf{( m A )}$ \\
\hline-20 & 5.21 \\
\hline-10 & 6.10 \\
\hline 0 & 8.47 \\
\hline+6 & 13.77 \\
\hline+10 & 21.48 \\
\hline
\end{tabular}

Table 1. Current consumption measured by Shnayder et al., 2004

The results shown in Table 1 were used to compute the energy consumption required by each transmission power level. Fig. 1 shows error-bar plots of radio and total energy consumption at $-20,-10,0,+6$ and $+10 \mathrm{dBm}$. An analysis of power usage and conservation with respect to the maximum power level is described in Table 2.

According to Fig. 1, several observations can be made. Firstly, an increase in transmission power results in a higher energy consumption. Transmitting data at lower power uses less energy. For example, over $75 \%$ of energy can be conserved if the minimum power is used for transmission instead of the maximum. Secondly, the radio unit consumes a significant amount of energy. Up to $56 \%$ and $84 \%$ of energy are used by the radio if the sensor transmits at minimum and maximum power levels, respectively. The results are validated by the CC1000 data sheet which is the employed radio in Mica2. According to the CC1000 datasheet, the required current consumption for -20 and $+10 \mathrm{dBm}$ are 6.9 and 26.7 milli-amp (mA), respectively. Therefore, over $74 \%$ can be conserved and this is close to the $75 \%$ which is obtained from PowerTOSSIM.

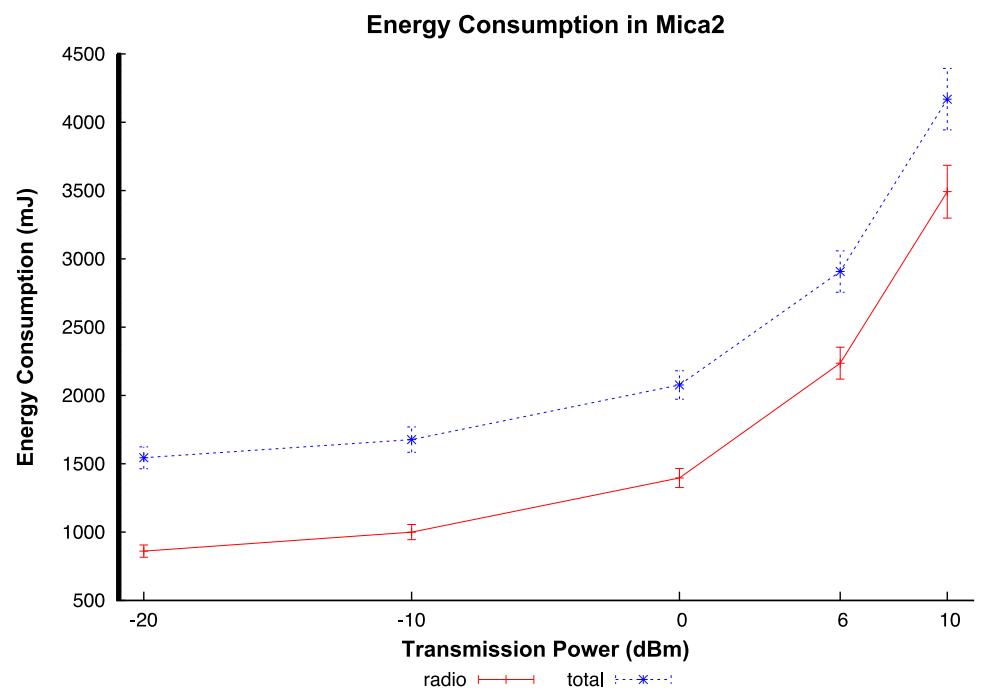

Fig. 1. Radio and total energy consumption at various transmission power levels 


\begin{tabular}{|c|c|c|c|}
\hline $\begin{array}{c}\text { Transmission Power } \\
(\mathbf{d B m})\end{array}$ & $\begin{array}{c}\text { Average of Radio } \\
\text { Power Consumption } \\
\mathbf{( m J )}\end{array}$ & $\begin{array}{c}\text { Percentage of Used } \\
\text { Power }\end{array}$ & $\begin{array}{c}\text { Percentage of Saved } \\
\text { Power }\end{array}$ \\
\hline-20 & 861.52 & 24.67 & 75.33 \\
\hline-10 & 1000.33 & 28.64 & 71.36 \\
\hline 0 & 1396.44 & 39.98 & 60.02 \\
\hline+6 & 2236.90 & 64.05 & 35.95 \\
\hline+10 & 3492.48 & 100 & 0 \\
\hline
\end{tabular}

Table 2. Average radio power consumption (mJ) and percentages of used and saved power

Two key motivations are established with respect to the simulation results. Firstly, transmission power considerably affects radio power consumption. The power-aware approach based upon power adaptation is Transmission Power Control (TPC). PoRAP adopts the TPC concepts in order to achieve the power conservation goal. The selected sensor platform in this work is Tmote and it employs the CC2420 radio instead of the CC1000. Like the CC1000, the CC2420 also supports transmission power adaptation but it provides a different range of power levels. Table 3 shows some of the possible power levels and the corresponding current consumption. An analysis of power conservation with respect to the maximum level is also shown.

\begin{tabular}{|c|c|c|c|}
\hline $\begin{array}{c}\text { Transmission Power } \\
(\mathbf{d B m})\end{array}$ & $\begin{array}{c}\text { Current Consumption } \\
(\mathbf{m A} \mathbf{)}\end{array}$ & $\begin{array}{c}\text { Percentage of Used } \\
\text { Current }\end{array}$ & $\begin{array}{c}\text { Percentage of Saved } \\
\text { Current }\end{array}$ \\
\hline-25 & 8.5 & 48.85 & 51.15 \\
\hline-15 & 9.9 & 56.90 & 43.10 \\
\hline-10 & 11.2 & 64.37 & 35.63 \\
\hline-7 & 12.5 & 71.84 & 28.16 \\
\hline-5 & 13.9 & 79.89 & 20.11 \\
\hline-3 & 15.2 & 87.36 & 12.64 \\
\hline-1 & 16.5 & 94.83 & 5.17 \\
\hline 0 & 17.4 & 100 & 0 \\
\hline
\end{tabular}

Table 3. Transmission power levels provided by CC2420 and analysis of power conservation

According to Table 3, over $50 \%$ of power can be saved if the minimum power is used for data transmission. The transmission power is one of the main factors which produces different reception strengths. The power adaptation is based upon the current link quality in order to maintain a good link. However, power adaptation is based upon several factors affecting link quality such as distance and time-of-day.

Secondly, according to Fig. 1, the radio unit accounts for a significant amount of power compared to the total consumed by all hardware components. Keeping the radio in sleep mode after the sensor has transmitted the data may establish an enhancement in power conservation. This is feasible if the single-hop network sensors do not listen to transmissions from other nodes in order to discover optimal data paths. The schedule-based MAC (Medium Access Control) approach suits the direct communication scenario as each of the sources wake up for control reception and data transmission. Otherwise, they are in sleep mode and consume the least amount of communication energy. 


\subsection{Environmental investigation of transmission power and reliability}

This section provides details of experimental studies aimed at establishing effects of transmission power, distances and time-of-day on link quality metrics. In total three metrics including RSSI (Received Signal Strength Indicator), LQI (Link Quality Indication) and PRR (Packet Reception Rate) are used to describe the effects. The relationships between the metrics are also investigated and will be used for establishing power adaptation policies.

\subsubsection{Link quality metrics}

There is a variety of sources which cause variability in link quality in wireless communication. Unlike wired communication, environmental factors such as climatic conditions and time-ofday also affect the degree of signal attenuation. A significant degree of signal attenuation or interference may lead to unsuccessful data transmission. Link quality measurement is therefore one of the major issues in wireless network communication.

A transmitter sends data packets at a specific transmission power wirelessly over a medium to a receiver. The transmission power level is programmable and this capability is provided by a transceiver or radio unit which is a component responsible for data transmission and reception. A sensor communicates with the other node by sending and receiving messages via wireless channel which is normally air. Several signals are generated from various sources such as electronic appliances and they are dissipated to the air. A wireless channel may then have background noise which is capable of interfering with data delivery between a pair of nodes. Moreover, time-of-day and climatic conditions such as fog and rain affects the wireless link quality. In order to determine link quality characteristics, all causes of signal strength reduction are considered as sources of signal attenuation. The reduced magnitude in signal strength is therefore defined as signal attenuation. If the transmission power is less than signal attenuation, the message cannot be successfully received. When the receiver is not able to receive the sent packet and the number of received packets is not increased, the reliability requirement defined by an application may not be met. Transmission power should be adjusted in response to the changing link quality.

A radio unit provides several mechanisms to measure received signal power. The measured values are categorised as received signal strength (RSS). In total two attributes including RSSI (Received Signal Strength Indicator) and LQI (Link Quality Indication) are in the RSS category. The RSS can be used to indicate link quality. The reliability requirement specified by an application indicates a required number of packets received at the base station. The percentage of data receptions can be used to describe the link quality. The packet reception rate (PRR) is therefore introduced. Relationships amongst transmission power (TX), received signal strength (RSS) based attributes and PRR is useful for mapping application requirements to link quality measurements. Thus, the transmission power is adapted in order to provide reliability of packet reception.

Received Signal Strength Indicator (RSSI) is defined as a measurement of the signal strength of an incoming message. The transmitted signal strength or transmission power reduces as the signal propagates through the medium. The RSSI is measured at the receiver and it demonstrates the received signal strength. Therefore, signal attenuation is approximately the difference between the transmission power and the RSSI. Link Quality Indication (LQI) is another metric in the RSS-based category. According to the definition outlined in IEEE 802.15.4 Standard for Local and Metropolitan Area Networks, the LQI measurement is a characterisation of the strength and/or quality of received packet. Each received packet has its own LQI measurement and the integer value ranges from 0 to 255 . Therefore, the 
minimum and maximum values of LQI for each packet are 0 and 255, respectively. The IEEE standard recommends at least eight unique values of LQI should be used in order to yield a uniform distribution between the two limits. The following details of LQI are based upon the CC2420 radio unit as it is used in both Tmote Sky and Tmote Invent which are the chosen platforms in this research. Apart from RSSI and LQI, PoRAP determines an additional link quality index. The main reason is that both RSSI and LQI are not transparent to the user or application. Mapping mechanisms are required in order to convert an application requirement to the ranges of RSSI and LQI values the base station should have. This subsection aims to describe the Packet Reception Rate (PRR) which is more closely related to the application requirement. In this research, the PRR is defined as a percentage of the number of correctly received to that of transmitted packets. The PRR value is in the range of $0 \%$ to $100 \%$. The $100 \%$ PRR indicates complete reliability. Each received packet has its own measured RSSI and LQI which can be used to predict the PRR. Models representing relationships amongst metrics are therefore required and demonstrated later in this chapter.

\subsubsection{Experimental setup}

In our implementation-based experiments, Tmote Invent and Tmote Sky are used as the sensor and base station, respectively. Both of them employ the CC2420 radio which has working frequency band from 2,400 to 2,483 Megahertz (MHz). The radio transmission data rate is 250 kilobits per second (kbps). The random access memory (RAM) and program flash sizes are 10 and 48 kilobytes (Kbytes). The main difference between both platforms is that the Tmote Invent provides built-in sensor and battery boards. The minimum and maximum transmission power levels are -25 and $0 \mathrm{dBm}$, respectively. Tmote sensors consume 8.5 and 17.4 milli-amps (mA) for transmitting a data packet at minimum and maximum power levels, respectively. A current of $19.7 \mathrm{~mA}$ is required for radio receiving. This indicates that receiving accounts for a large radio power usage. Listening removal in PoRAP may enhance power conservation in WSN. Each Tmote sensor includes an internal Inverted-F antenna which is a wire monopole. The top section of the antenna is folded down to be parallel with the ground plane. The communication ranges for indoor and outdoor are $50 \mathrm{~m}$ and $125 \mathrm{~m}$, respectively.

The experiments were conducted in the $16 \mathrm{~m} \times 20 \mathrm{~m}$ indoor environment. The base station was plugged into a desktop computer and received data from sensors. Three sensors were used and they were placed at the same locations. In total 10 locations including 1, 2, 3, 4, 5, 7, $10,13,16$ and $20 \mathrm{~m}$ were used. The sensors and base station had the same antenna orientation and height above floor level. The payload size was 12 bytes. In total 8 transmission power levels including 3, 7, 11, 15, 19, 23, 27 and 31 associated to -25, -15, -10, $7,-5,-3,-1$ and $0 \mathrm{dBm}$ were used. The sensors transmitted one packet every second. At each power, the sensors transmitted 50 packets for statistical analysis. Upon data reception, the base station measured RSSI and LQI. The number of received packets was counted in order to compute PRR.

\subsubsection{Experiments on location as a determination of necessary transmission power}

The significance of the locations of the sending and receiving motes to determine the relationship between transmission power (TX) and reception quality is established. In this experiment, the base station location was the same whilst three sensors were placed at 10 different locations in the same direction with clear line-of-sight (LOS) including 1, 2, 3, 4, 5, 7, 10, 13, 16 and 20m. Each power adaptation cycle was ended after the maximum power 
had been reached. The other experimental parameters such as power levels, data sending rate and number of runs are stated in Section 4.2.2.

Fig. 2 shows the average RSSI readings of the three sensors at various locations and transmission power levels. The missing data indicate that the power provides RSSI reading less than $-95 \mathrm{dBm}$ which is the minimum value reported by TinyOS. Fig. 3 shows average LQI readings of a sensor at various locations and transmission power levels. The missing data indicate unsuccessful data delivery.

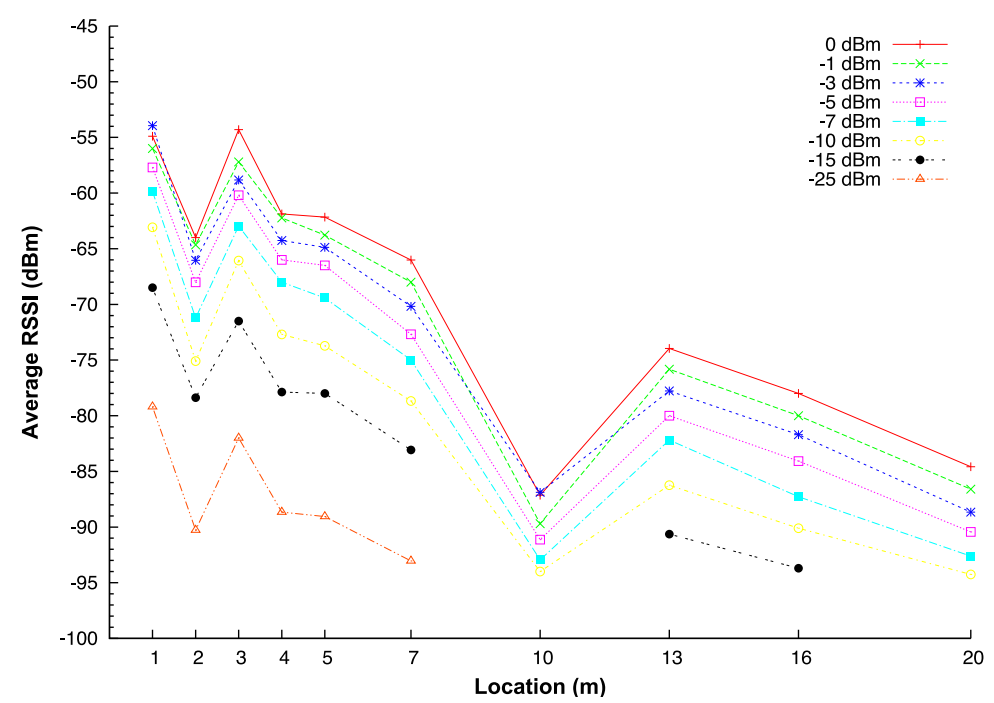

Fig. 2. Effects of sensor locations on RSSI

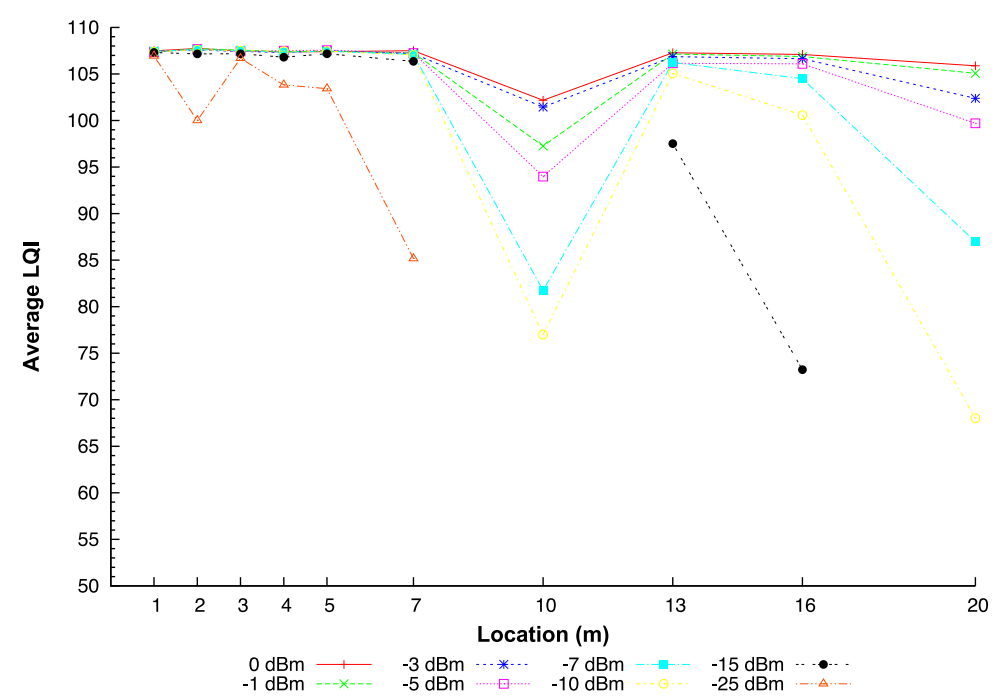

Fig. 3. Effects of sensor locations on LQI 
According to Fig. 2 and Fig. 3, most of the RSSI measurements proportionally increased with the transmission power levels. Unlike the RSSI, the LQI measurements were stable at closer locations especially when higher power was used for transmission. Most of the LQI values decreased at greater distances. The minimum power level of $-25 \mathrm{dBm}$ could be used to successfully deliver data to the base station only when the locations were within $7 \mathrm{~m}$. The decrease in received signal strength with increasing distances assumed in the prediction models do not apply in the results. For example, in the case of $2 \mathrm{~m}$, the sensor provides a weaker strength compared to a distance of $3 \mathrm{~m}$. The experimental results given in (Lin et al., 2006) and (Stoyanova et al., 2007) demonstrate similar observations on location effects. The RSSI and LQI are measured only when the base station receives data. The observed minimum RSSI values higher than $-95 \mathrm{dBm}$ indicate data reception.

\subsubsection{Fluctuation in link quality metrics over time of day}

This section investigates on how RSSI, LQI and PRR fluctuate over the time of day. The same base station and Sensor 1 were used. The sensor was located at $20 \mathrm{~m}$ in the same environment. It transmitted one packet every second at $0 \mathrm{dBm}$ for 1,440 minutes or 24 hours. The experiment was started in the morning before the office hour.

Fig. 4 demonstrates fluctuation of the RSSI, LQI and PRR over time of day. The RSSI fluctuated during the first half of the experiment. It was stable during the night time and the fluctuation was back later in the experiment. Unlike the RSSI, the LQI fluctuated throughout the experiment. At the beginning the PRR siginificantly decreased. This observation was resulted from the presence of people around the lab. The PRR increased during the night time as there were no staff and student in the lab.

In summary, apart from transmission power, location and heterogeneity in the manufacture, the link quality metrics are affected by the time-of-day. The presence of people around the lab is the main factor in this experiment and is considered as temporary physical barrier. Radio communication in WSN requires a line-of-sight. Some packets may be lost if there are some people in the sending path.

\subsubsection{Relationship between metrics}

This section aims to describe the relationships between RSSI, LQI and PRR. During packet reception, the base station measures RSSI and LQI. Apart from RSSI and LQI, the standard message type of TinyOS includes the CRC field which is a Boolean data type. The base station also looks at the CRC field to see if the data packet is received correctly. The numbers of data transmissions and receptions are counted to compute the PRR. This scheme can be used in a long-term operation.

However, the PRR may be estimated from the RSSI or LQI measurements. This concept suits a short term operation. The base station does not count the numbers of sent and received packets. Hence, the relationship between metrics needs to be established. Fig. 5 shows relationships between the link quality metrics at $5 \mathrm{~m}, 12 \mathrm{~m}$ and $19 \mathrm{~m}$. The average RSSI and LQI are computed at each transmission power level. The number of received packets is counted in order to calculate the PRR.

According to Fig. 5, several observations can be made as follows:

1. The PRR steeply increases with RSSI up to a certain point followed by more stable reliability measurements. Significant variations in reception rates are found when the RSSI readings are between -95 and $-90 \mathrm{dBm}$. At least 95\% PRR may be achieved at all distances if the sensor transmits data at the power producing RSSI greater than $-90 \mathrm{dBm}$. 
2. The higher LQI results in a more stable PRR. The relationship between LQI and PRR shown in Fig. 5 (b) is less clear than Fig. 5 (a). Similar results are also addressed in (Lin et al., 2006). According to these observations, RSSI should be used to relate to the PRR.

3. The LQI significantly increases with the RSSI. Convergence to particular LQI values is then observed. A lower bit error rate is observed when the base station receives packets with higher RSSI measurements.

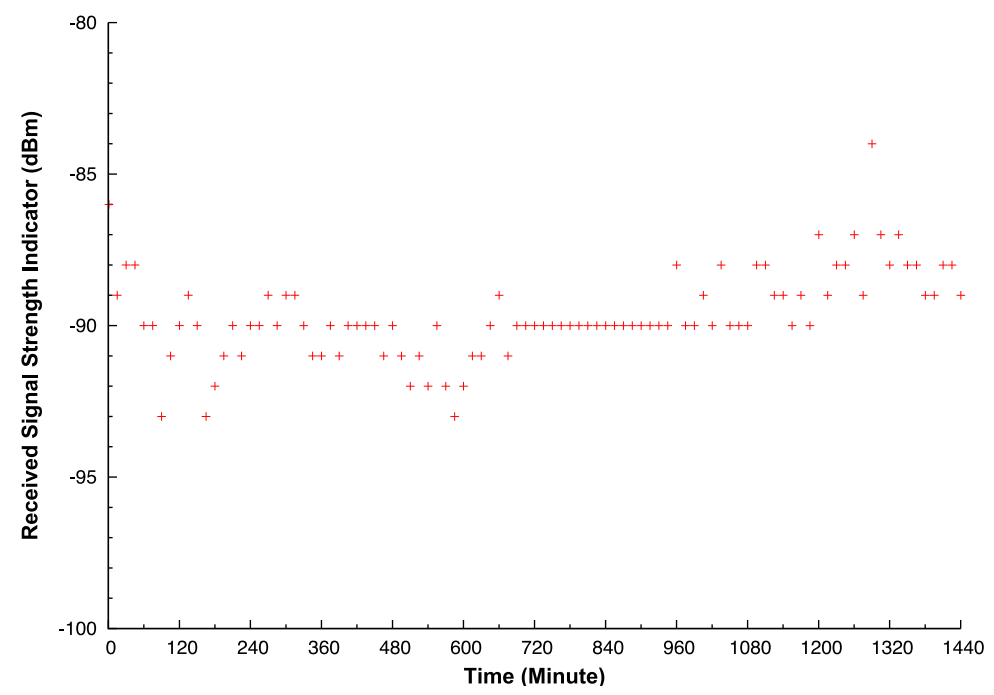

Fig. 4. (a) Fluctuation in link quality metrics over 24 hours RSSI

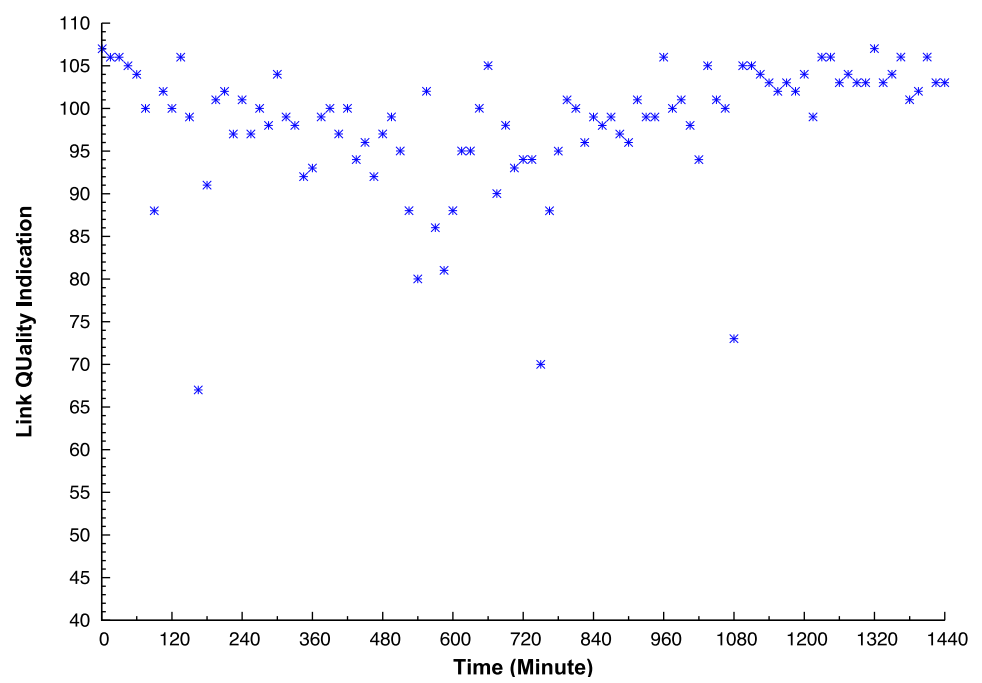

Fig. 4. (b) Fluctuation in link quality metrics over 24 hours LQI 


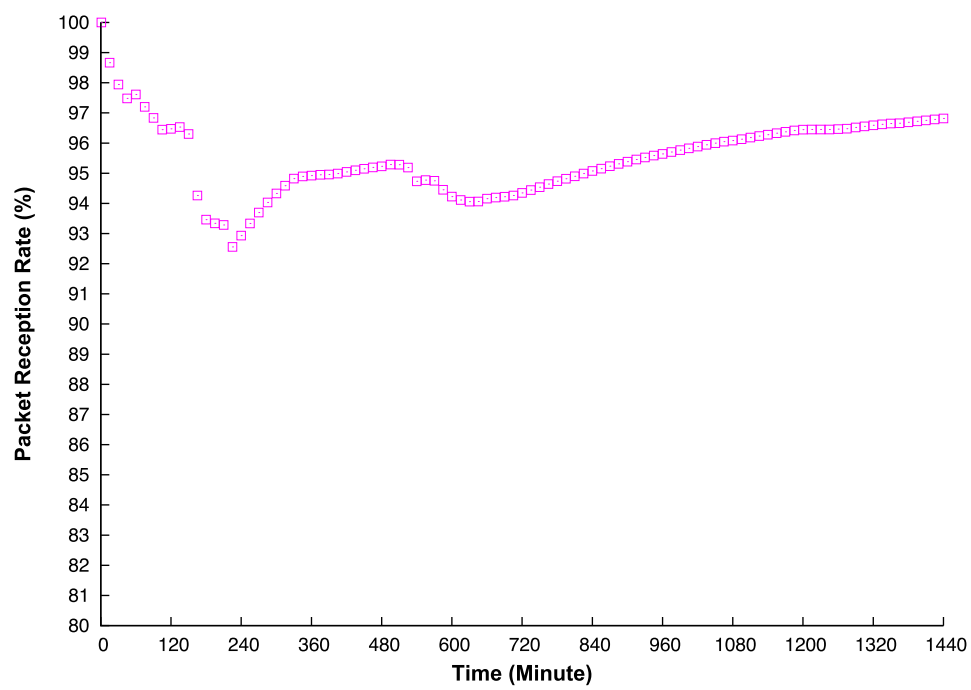

Fig. 4. (c) Fluctuation in link quality metrics over 24 hours PRR

The relationship between link quality metrics can be used to estimate an observed reliability from the measured receiving strength. This observation is addressed in (Lin et al., 2006) and (Srinivasan et al., 2006). After measuring the metrics, the base station determines whether the current transmission power requires an adaptation. The PRR steeply increases with the RSSI followed by significantly more stable measurements. The PRR should not be estimated from the RSSI between -95 to $-90 \mathrm{dBm}$ as transmission power adaptation based upon this region will not be accurate. The measurements demonstrate that the network should operate at levels taken from an appropriate region.

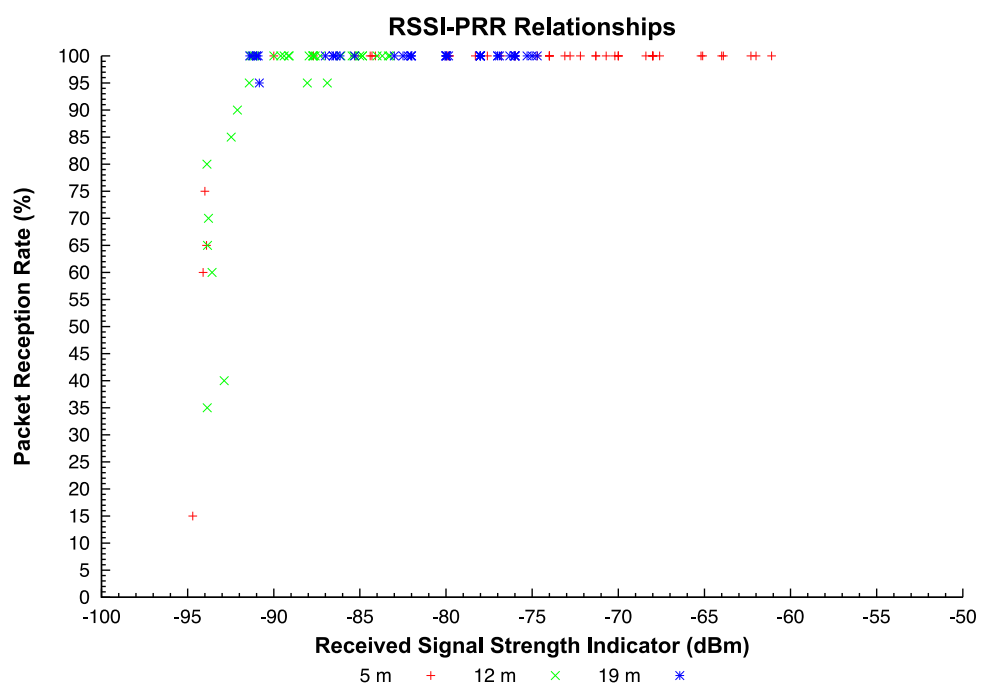

Fig. 5. (a) Relationships between metrics RSSI-PRR 


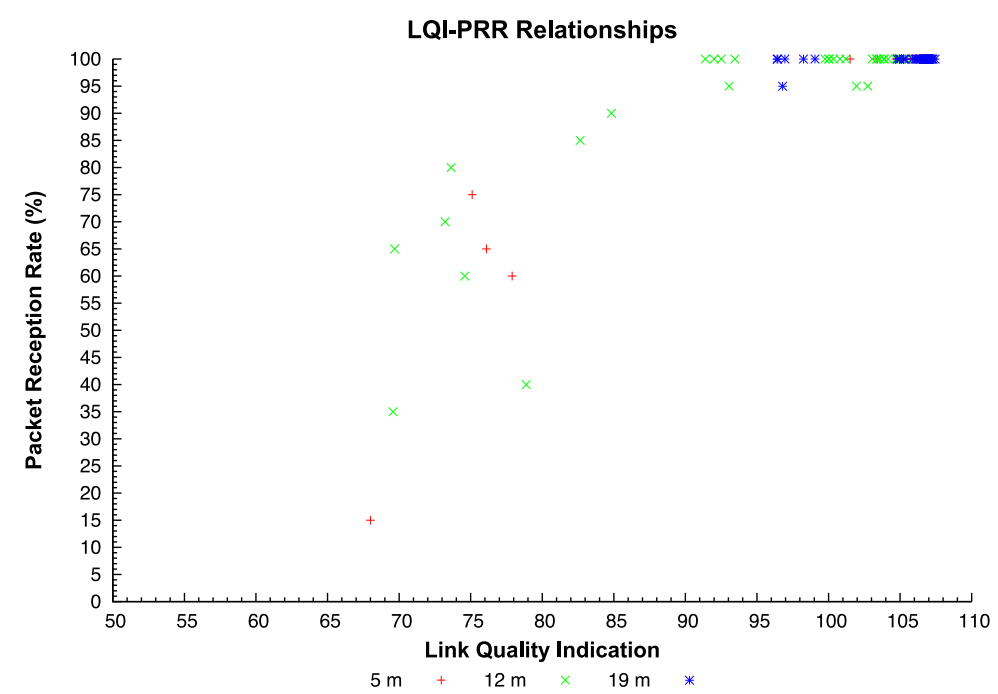

Fig. 5. (b) Relationships between metrics LQI-PRR

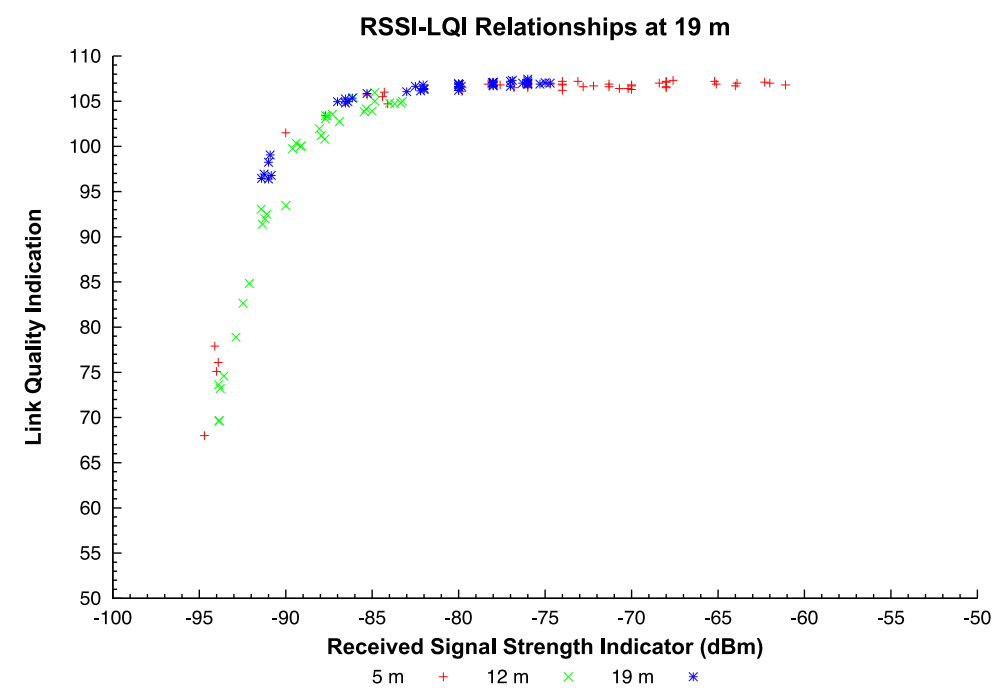

Fig. 5. (c) Relationships between metrics RSSI-LQI

\subsection{Delays in wireless sensor network}

This section provides some experimental results on delays in wireless sensor network (WSN) which affects PoRAP architecture development. Communication is represented by a frame structure which consists of several slots. A slot is assigned to each source and it transmits data when the allocated slot arrives. The slot length should be long enough to avoid data collisions at the base station where two packets from two different sources arrive approximately at the same time. Several experiments have been conducted in order to 
investigate some factors which affect the delays, including heterogeneity in sensor manufacturing and payload sizes.

\subsubsection{Timestamp measurements and delay calculations}

Details of timestamping scenario and delay calculations are given. As the base station does not know when the source is booted, at the beginning it broadcasts the control packet periodically. The periodic broadcast was set to 1 second. After the source is booted, it starts its transmission after the packet has been received. Similarly, the base station starts the next transmission after it has received the packet back from the source. Packet timestamping mechanisms and delay calculations are respectively illustrated in Fig. 6 and Table 4.

According to Fig. 6 , the base station is booted at $x_{0}$. When the base station is ready to send, the timer is set to be fired at $x_{1}$ and send command is called at $x_{2}$. A timer is used in order to trigger packet transmission. Prior to transmission, the base station sets some fields in the message structure such as its id and transmission power. The SFD (Start of Frame Delimiter) transmission occurs at $x_{3}$. The timestamp is created and the packet payload content is modified to include the time of the transmission. Therefore, the fire-to-send and send command delays of the base station are equal to $x_{2}-x_{1}$ and $x_{3}-x_{2}$. The packet is completely transmitted by the radio at $x_{4}$ and the transmission delay is $x_{4}-x_{3}$.

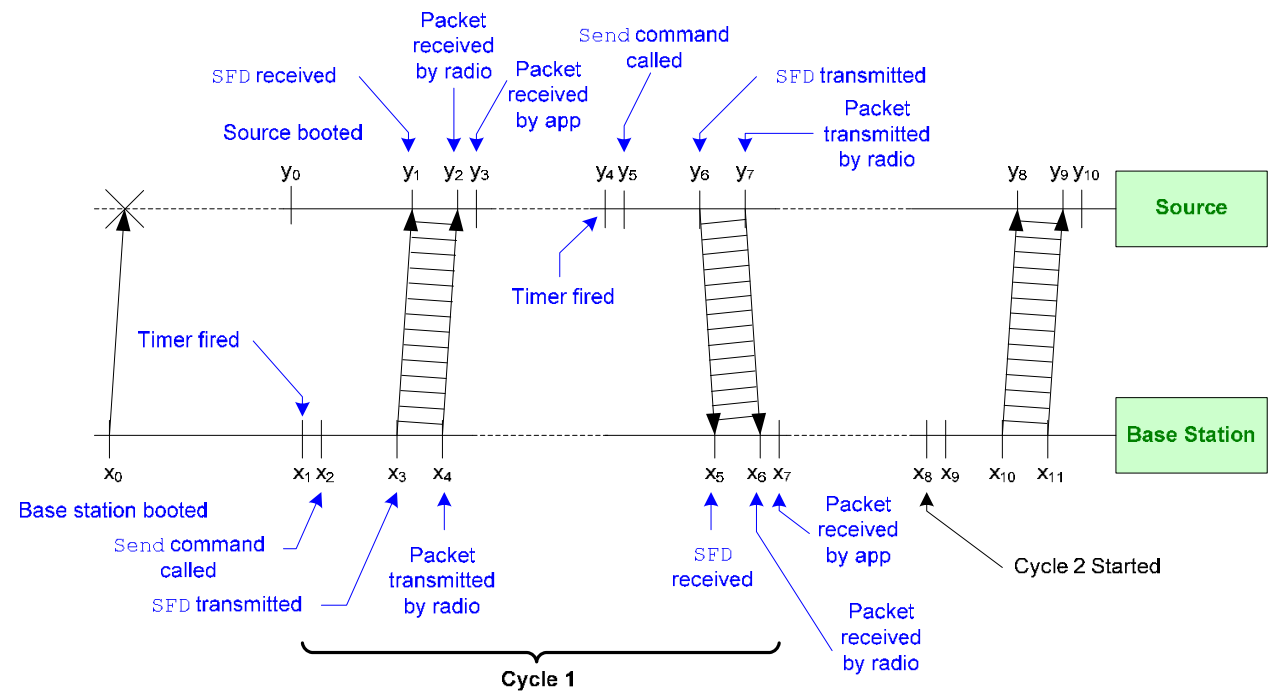

Fig. 6. Timestamp at various events

After being booted at $y_{0}$, the source receives the SFD at $y_{1}$. The receive event of the radio and application are signalled at $y_{2}$ and $y_{3}$ when the source receives the packet. The reception and receive delays of the base station are therefore $y_{2}-y_{1}$ and $y_{3}-y_{2}$. Once the packet has been received, the source requires some duration to process the information obtained from the packet. It then sets up its own transmission and the bits of packet are loaded into the radio buffer. The timer is fired at $y_{4}$ and the send command is called at $y_{5}$. The SFD is transmitted at $y_{6}$. Hence, the send command delay of the source is equal to $y_{6}-y_{5}$. The transmission delay is $y_{7}-y_{6}$. Table 4 summarises the delay calculations. 


\begin{tabular}{|c|c|}
\hline Delays & Calculations \\
\hline \multicolumn{2}{|l|}{ Base Station } \\
\hline - $\quad$ Fire-to-Send & $x_{2}-x_{1}$ \\
\hline - $\quad$ Send Command Delay & $x_{3}-x_{2}$ \\
\hline - $\quad$ Transmission & $x_{4}-x_{3}$ \\
\hline - $\quad$ Reception & $x_{6}-x_{5}$ \\
\hline - $\quad$ Receive & $x_{7}-x_{6}$ \\
\hline \multicolumn{2}{|l|}{ Source } \\
\hline - $\quad$ Reception & $y_{2}-y_{1}$ \\
\hline - $\quad$ Receive & $y_{3}-y_{2}$ \\
\hline - $\quad$ Fire-to-Send & $y_{5}-y_{4}$ \\
\hline - $\quad$ Send Command Delay & $y_{6}-y_{5}$ \\
\hline - $\quad$ Transmission & $y_{7}-y_{6}$ \\
\hline Two-Way Propagation & $\left(x_{5}-x_{3}\right)-\left(y_{6}-y_{1}\right)$ \\
\hline
\end{tabular}

Table 4. Summary of delay calculations

According to Table 4, the transmission and reception delays are calculated based upon when the events take place. The transmission delay is defined as the duration required for the radio to transmit the packet. In TinyOS 2.x the CC2420Transmit interface provides a sendDone() event which notifies packet transmission completion. The reception delay is the duration required for packet reception by the radio, and the receive event is used for the timestamp. The fire-to-send delay indicates the desired interval for starting packet transmission after the timer is fired.

One Tmote Sky base station and one Tmote Invent source were used. The source was located at $0.5 \mathrm{~m}$ away from the base station. The base station was plugged into a desktop computer. In total 1,000 cycles of message exchange were run for each source. After the packet had been received, the node waited for $128 \mathrm{~ms}$ and initiates its data transmission.

\subsubsection{Experimental results}

In order to consider the effects of payload size, an additional experiment was conducted. The scenario shown in Fig. 6 was used. All settings are the same except the payload sizes. In total five payload sizes were used including 39, 55, 75, 95 and 115 bytes. Note that the maximum payload for the CC2420 radio is limited to 117 bytes whilst the header size is 11 bytes. Send command and transmission delays of the source were determined. Two-way propagation delays were also computed. In the case of 39 bytes, reception and receive delays of source and base station were observed whilst all delays were observed for the larger payload sizes.

Statistical analysis of fire-to-send, send and transmission delays in milliseconds were conducted. The relationships between the $50^{\text {th }}$ percentiles or medians of all sending delays and payload sizes are shown in Fig. 7. Note that "Send Command" delay is represented as "Send" in the figure. The results show that all delays increase with increasing payload sizes. The source requires more time to deliver larger packets to the radio. Similarly, larger packets require a longer duration for transmission. Increases in send command and transmission delays are greater than those of fire-to-send delay.

Statistical analyses of reception and receive delays in milliseconds were also made. The relationships between the $50^{\text {th }}$ percentiles or medians of both receiving delays and payload sizes are shown in Fig. 8. Linear relationship between reception delay and payload size is also observed in Fig. 8. The receive delays are constant for all payload sizes. 
The 32-KHz clock has been used in this experimental study and provides 32,768 ticks per second. There are 32 ticks in one millisecond. Therefore, the finest precision is approximately 0.03125 millisecond or 31.25 microseconds. The two-way propagation delays for all payload sizes are calculated and frequencies of the delay occurrences in ticks are shown in Table 5.

According to Table 5, frequencies of the 0-tick decrease with increasing payload sizes. Larger packets require more time to travel from source to destination. However, the twoway propagation delays are significantly less than the other delays.

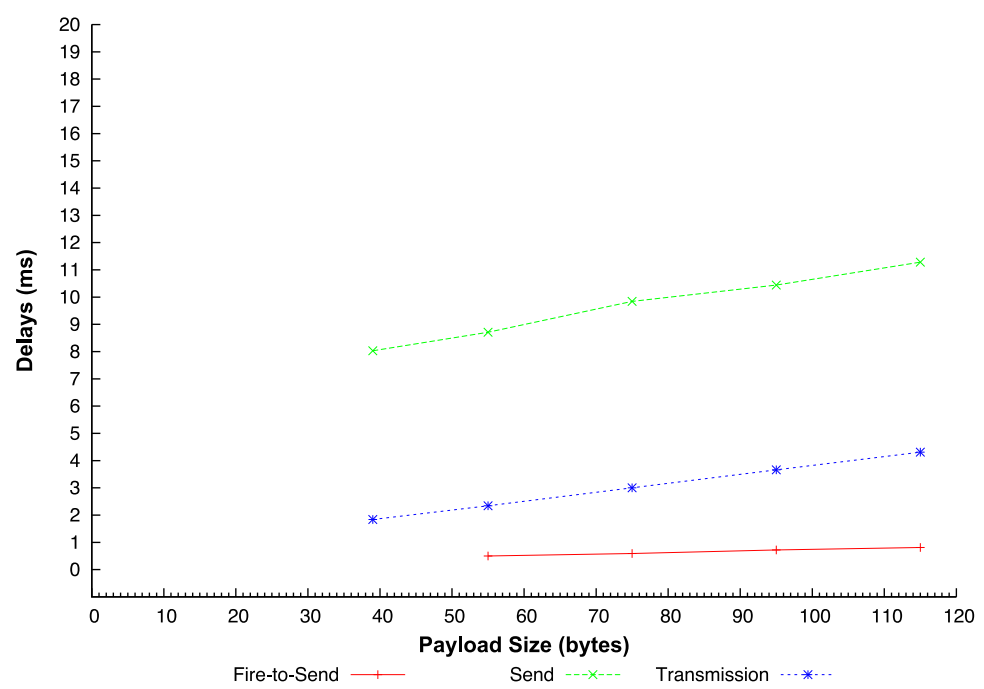

Fig. 7. Relationships between source sending delays and payload sizes

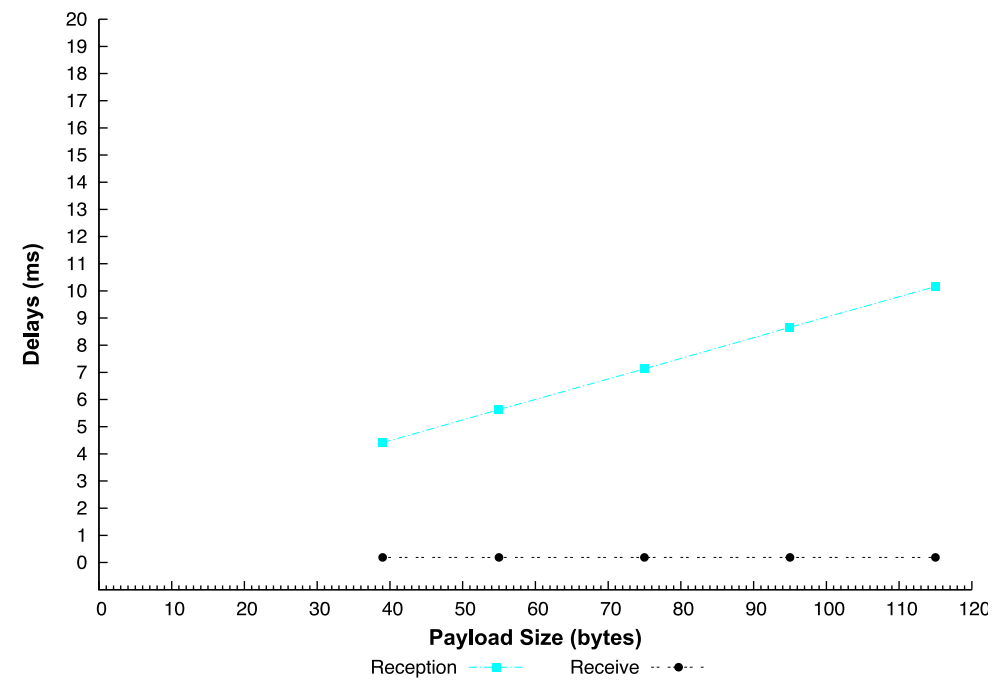

Fig. 8. Relationships between source receiving delays and payload sizes 


\begin{tabular}{|c|c|c|c|c|c|c|}
\hline \multicolumn{2}{|c|}{ Attribute } & \multicolumn{5}{c|}{ Payload Size (bytes) } \\
\cline { 3 - 7 } & 0 & 39 & $\mathbf{5 5}$ & $\mathbf{7 5}$ & $\mathbf{9 5}$ & $\mathbf{1 1 5}$ \\
\hline \multirow{3}{*}{ Frequencies } & 1 & 141 & 193 & 212 & 245 & 259 \\
\cline { 2 - 7 } & 2 & 0 & 0 & 3 & 0 & 0 \\
\cline { 2 - 7 } & 2 & 999 & 1,000 & 1,000 & 1,000 & 999 \\
\hline \multicolumn{2}{|c|}{ Cycles } &
\end{tabular}

Table 5. Frequencies of two-way propagation delays

\section{Design of PoRAP}

This section describes the design of PoRAP (Power \& Reliability Aware Protocol) which aims at minimising communication energy in wireless sensor network (WSN). The experimental results stated in previous section inform the design.

\subsection{PoRAP main capabilities}

In PoRAP, power can be conserved via transmission power adaptation and efficient medium access management. The selected link quality index is Received Signal Strength Indicator (RSSI) and it is measured by the base station during data reception. Along with the awareness of data loss, the adjusted power will often maintain the network operating at the region where data loss is minimised.

Additional communication can be saved by adopting the schedule-based MAC approach. Sending and receiving delays can be estimated as they are dependent upon packet size whilst two-way propagation delay is significantly small. Data transmissions are scheduled and the sources are mostly in sleep mode to conserve energy. Only one source engages the shared medium at a time for data transmission. Thus, data collision can be avoided and idle listening can be minimised. More explanations on PoRAP key capabilities are given as follows:

\subsubsection{Schedule-based protocol}

In the single-hop networks, sources are capable of communicating with their base station directly. This scenario is feasible when the sources and base station are located within communication range of each other. The base station may be connected to several sensors which require an access to the shared medium. Uncontrolled medium access possibly leads to data collisions at the base station. Collision is one of the main sources of power wastage in the WSN shared medium system. The medium access control (MAC) approach attempts collision avoidance. There are currently two main approaches proposed for WSN. Firstly, the medium is sensed to detect any ongoing activities in the medium before conducting data transmission and reception. This scheme is named contention-based.

PoRAP employs another approach in which each node is assigned a specific duration to use the shared medium. This scheme is called schedule-based. The other sensors cannot access and use the medium whilst a sensor is communicating within its time slot. Sources listen to the base station only once in a frame. Idle listening is therefore minimised. Moreover, data collisions at the base station can be avoided as there is only one source sending at a time. The slot length should be long enough to let the source and base station complete data transmission and reception. This scheme may not be suitable in the case of multi-hop WSN where each resource-constrained sensor has to maintain slot information 
of its neighbours. Furthermore, time synchronisation is required as both sender and receiver have to orchestrate the data communications to avoid collision caused by the other receivers.

Centralised scheduling control by the base station is feasible in PoRAP. Slot arrangement information can be sent to all sensors located in the range. The base station broadcasts a packet to all sources located in its range. Slot information such as number of slots, slot length and start time of first slot are included in the payload. Once the first frame is finished, the base station broadcasts again with the transmission power adaptation notification.

\subsubsection{Communication power conservation}

Power constraint should be taken into account when designing a protocol for WSN. Sensors may be left unattended after being deployed in the remote or hostile environment where battery recharge or replacement may be costly or infeasible. Communication accounts for power consumption in WSN. Several sensor platforms provide adaptation to the transmitting power and the concept of Transmission Power Control (TPC) has been adapted to WSN. The CC2420 radio employed by Tmote platform, which is used in this research, supports transmission power (TX) setting. The TX levels are stated by a 5-bit number. There are therefore 32 possible TX settings provided by the CC2420. In TinyOS, the setPower() command provided by CC2420Packet interface accepts a value between 0 to 31 for TX setting. However, the CC2420 datasheet specifies programmable TX in 8 steps from approximately -25 to $0 \mathrm{dBm}$ which are respectively equivalent to the power levels of 3 and 31. The Tmote datasheet follows guidelines given by the CC2420.

Transmission power adaptation policies in WSN should take application specifics into account. Different applications may require the sources to transmit data at different rates. For example, an environmental monitoring system may require the current temperature hourly whilst a surveillance system may require the data every second when an intrusion is detected. The sensors should be switched to sleep mode after transmission in order to minimise the idle listening. In a multi-hop network, each node is responsible for routing. It has to communicate with its neighbours to discover the best path by means of the least power utilisation. An amount of power is therefore required for listening in the multi-hop. However, a sensor in the single-hop scenario is capable of transmitting data directly to the base station. It may be switched to sleep mode after transmission. However, the source has to listen during the control slot transmission from the base station.

The power adaptation mechanisms in PoRAP do not require historic entries of RSSI and associated transmission power. The main reason is the limitation of buffering capacity of the radio chip. The base station should support a significant number of sources. In the CC2420 radio, the maximum buffer size is 128 bytes. Some bytes are required for the header and other controlling details. Only two bits are used to notify the power adaptation. The RSSIPRR relationship obtained from the experimental studies is considered for adaptation as it suggests the operating region for WSN. In the case of power adaptation, the base station sets particular bits to notify the source. The sources get the bits and set their transmission power accordingly.

\subsubsection{Link quality monitoring}

Radio communication uses air as the transmission medium. There are several attributes ranging from differences in hardware components to environmental factors such as physical 
barriers which affect signal attenuation. Received signal strength estimation is unlikely as sensors can be placed in various areas of interest. An estimation model should not only determine distance between sender and receiver as an input, location should also be taken into account. A shorter distance may not always provide a higher received strength if a physical barrier appears in the communication line-of-sight (LOS). Moreover, the link quality metrics fluctuate over the time of day. The observed strength in an indoor environment may be lower during the nighttime. Applying the simple received signal strength estimation models, focusing mainly on distance and hardware properties, may not be sufficient. Therefore, PoRAP employs the measurement-based approach in order to more accurately adapt the transmission power.

Two link quality metrics are used in PoRAP. The RSSI is obtained by the radio chip whilst the PRR is specified by the applications. The relationship between RSSI and PRR can relate the application requirement to the observed link quality. As shown in Section 4.2.5, a clear relationship between the two metrics is established. The PRR steeply increases with the RSSI up to a certain point. The PRR is then stable after a certain value of RSSI and a lower RSSI or TX can be used to obtain the required PRR.

The range of required RSSI is obtained from the reliability requirement and the RSSI-PRR relationship. This range is recognised by the base station. Upon data reception, the base station measures the RSSI and compares it to the RSSI thresholds. The adaptation bits are set with respect to the comparison result. There are three available patterns of bit settings; the transmission power will be increased if the measured RSSI is lower than require and it will be decreased if the RSSI is higher. The sources will be notified to retain the current power if the RSSI is within the range.

\subsection{PoRAP architecture}

This section aims to describe PoRAP architecture. PoRAP aims at an efficient data delivery in WSN by means of energy conservation. Input of PoRAP comes from two external components, the user/application and the monitored phenomenon. PoRAP recognises the duty cycle and the awareness of data loss. The sensed data is another input and it will be sent from the source to the base station. In order to achieve the goals, the base station controls the sources whereas the sources send data to the base station. Required functionalities of the base station and the sources are then stated. The interactions between them are described and they are used to address the required components within the source and the base station. Moreover, the interactions between such components are also given in this section.

\subsubsection{Overview of PoRAP}

The main objective of PoRAP development is to provide an efficient data communication in WSN where the user/application has his/its own requirements such as reliability and duty cycle. The development of a generic network protocol for WSN is challenging as WSN are application specific. Fig. 9 shows an overview of PoRAP architecture in terms of the interactions between its main components.

According to Fig. 9, four main components are addressed including the user/application, sensed phenomenon, base station and sources. As WSN is application specific, the user/application has its own set of requirements. The base station directly interacts with the user/application whilst the sources collect physical directly from the phenomenon. The functionalities required at the base station and source can be listed as follows: 


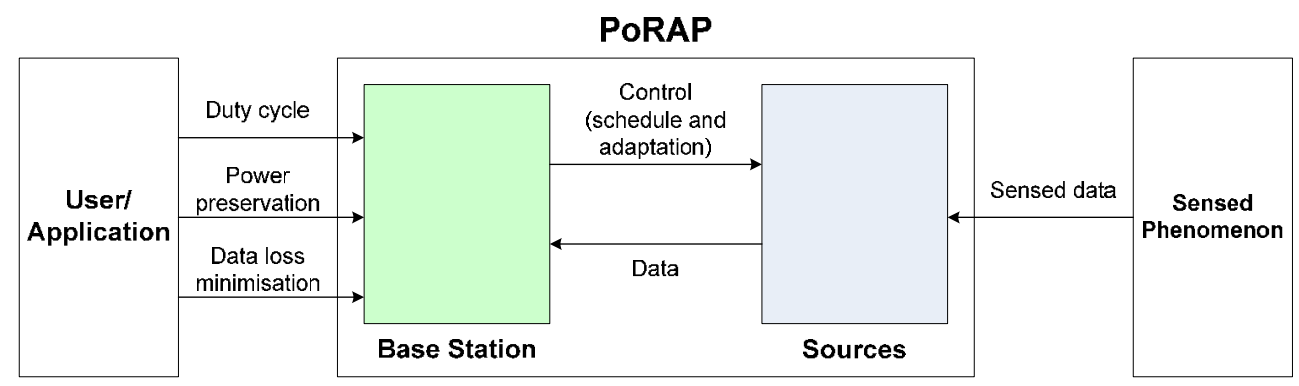

Fig. 9. Overview of PoRAP

\section{Base station:}

- Recognise the requirements of user/application: PoRAP aims at the low duty cycle application where the key objective is power conservation instead of throughput. Examples of this application category are habitat and environmental monitoring systems.

- Control the source's operation: This work focuses on the single-hop network where direct communication between sources and base station is feasible. No routing is required at each source and its operation is controlled by the base station in two aspects. Firstly, the base station determines whether transmission power used by the source needs to be adjusted by looking at the RSSI. Secondly, the communication cycle of each source is scheduled in order to avoid data collision and minimise idle listening.

\section{Source:}

- Collect physical data: WSN has been deployed to collect physical data from the targeted environment such as temperature and humidity. This work looks at how an efficient data delivery can be achieved by using lower transmission power whilst data loss is minimised. The processes of data collection are outside the scope of this study.

- Data transmission: After receiving the control information, the source sets two parameters. Firstly, it synchronises the communication schedule. Thus it will know when to start the radio for control reception and data transmission. Secondly, the source adapts its transmission power level according to the included notification. Lower power can be used and a significant amount of transmission power can be conserved.

Several interactions between the source and base station are required to achieve the functional requirements and they are addressed in Fig. 10.

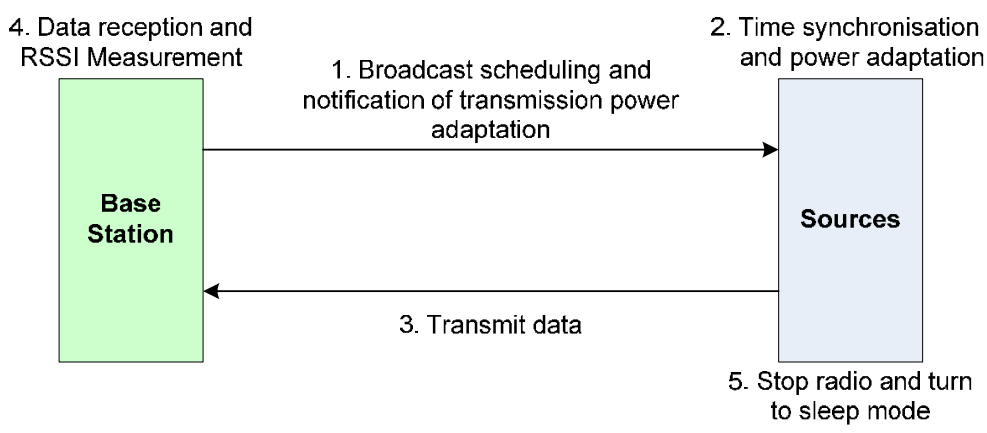

Fig. 10. Interaction between sources and base station 
1. PoRAP focuses on the set of fixed sources which are located within communication range of the base station. The control packet includes scheduling and power adaptation notification and is broadcast to the sources using the maximum power level. This is feasible as the base station obtains extra power from the connecting computer.

2. Once the control packet is received by the source. Information on scheduling and notification is read. The source synchronises its schedule with the other nodes together with adjusting its transmission power accordingly.

3. After conducting time synchronisation and transmission power adaptation, the source waits for its slot to conduct data transmission using the adjusted transmission power. The radio must be started for communication.

4. The base station measures the RSSI during data reception. The observed RSSI is compared to the desired range which includes minimum and maximum values. The setting of the RSSI thresholds is obtained from the RSSI-PRR relationship. The selected RSSI should be obtained from the region where significant stability in the PRR is observed. The base station then decides whether transmission power adaptation is required. The notification is set accordingly.

5. The source stops its radio after transmission to save power. The amount of power consumption is the least when the source is in sleep mode. Timing is required for the source to start the radio again for the next communication cycle.

\subsubsection{Components}

The previous section points out several essential functions which are required to achieve the objectives of PoRAP development. This section aims to describe the essential components which give rise to this functionality. The selected operating system for WSN in this work is TinyOS which already provides several useful components and PoRAP takes those in TinyOS and adds some further modifications. The main components are determined from the interactions including the user/application, the observed phenomenon, the base station and source. Several components required at the base station and source are then considered. Moreover, the interactions between each component are demonstrated.

\section{A) Components at base station and sources}

The base station recognises the requirements of the user/application and controls the sources based upon the requirements. As PoRAP aims at the direct communication, the control information is broadcast to the sources which are located within the communication range. After physical data collection, the sources set their communication parameters prior to data transmissions. Fig. 11 depicts several components required at the base station and sources.

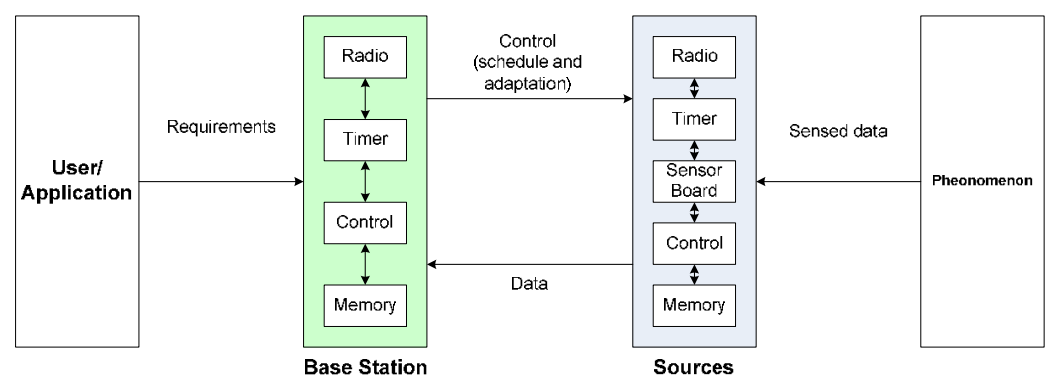

Fig. 11. Components at base station and sources 
Each of the required components is described as follows:

- Radio: Each sensor employs the radio communication for wirelessly communicating with its neighbours or destinations. The radio has four major functions as follows:

- Data communications: Control information is sent by the base station's radio chip and is received by the source's radio chip. Data is sent by the source's radio chip and is received by the base station's radio chip.

- Data buffering: Prior to forwarding the received data to the higher layers or transmitting the data through the medium, the data is buffered. The buffering capacity is limited and dependent upon the radio chip. The capacity is important to the design of packet structures. For example, the control packet must not be longer than the allowable capacity but it has to carry all the required information.

- Received signal strength measurement: The received signal strength is important as it can reflect the current link quality. The latest radio chip provides the measurement of received signal strength such as Received Signal Strength Indicator (RSSI) and Link Quality Indication (LQI). RSSI is used in this work as it can be obtained from several radio models and its relationship with the Packet Reception Rate (PRR) is clear.

- Transmission power adaptation: The RSSI changes with transmission power and several factors such as location, time-of-day and environment. One of the main features in PoRAP is transmission power adaptation. The key concept is adjusting the current transmission power to achieve the power conservation and data loss minimisation. The latest radio model supports programmable transmission power.

- Timer: WSN is considered a share-medium system as all nodes have to access the medium prior to transmission. PoRAP aims at single-hop WSN where direct communication between source and base station is feasible. The sources are not responsible for routing. Instead of applying the contention-based scenario, the transmissions are scheduled. A slot is allocated for each source so that it can send only when its slot arrives. Otherwise, the radio is stopped and the source is switched to sleep mode for minimum energy consumption. A timer is therefore required for scheduling the radio start and stop.

- Control: It is used to control the other components especially when there is no control mechanism provided for some components. For example, an additional control interface is required for the radio and the interface is used to start and stop the radio.

- Memory: This component is the basic one which is also included in the sensor. Several variables along with their values and measurements are stored in the memory. For example, the required RSSI range which is obtained from the RSSI-PRR relationship. This range is stored in the memory and will be compared to the observed RSSI to determine whether any transmission power adaptation is required.

- Sensor board: This component is crucial for the sensors as it is responsible for collecting the physical data from the environment. The sensor board consists of several sensors such as temperature and humidity.

B) Interactions between components

This section aims at addressing the interactions between the components, and they are described in Fig. 12. The interactions within the base station and source can be separately described as follows: 


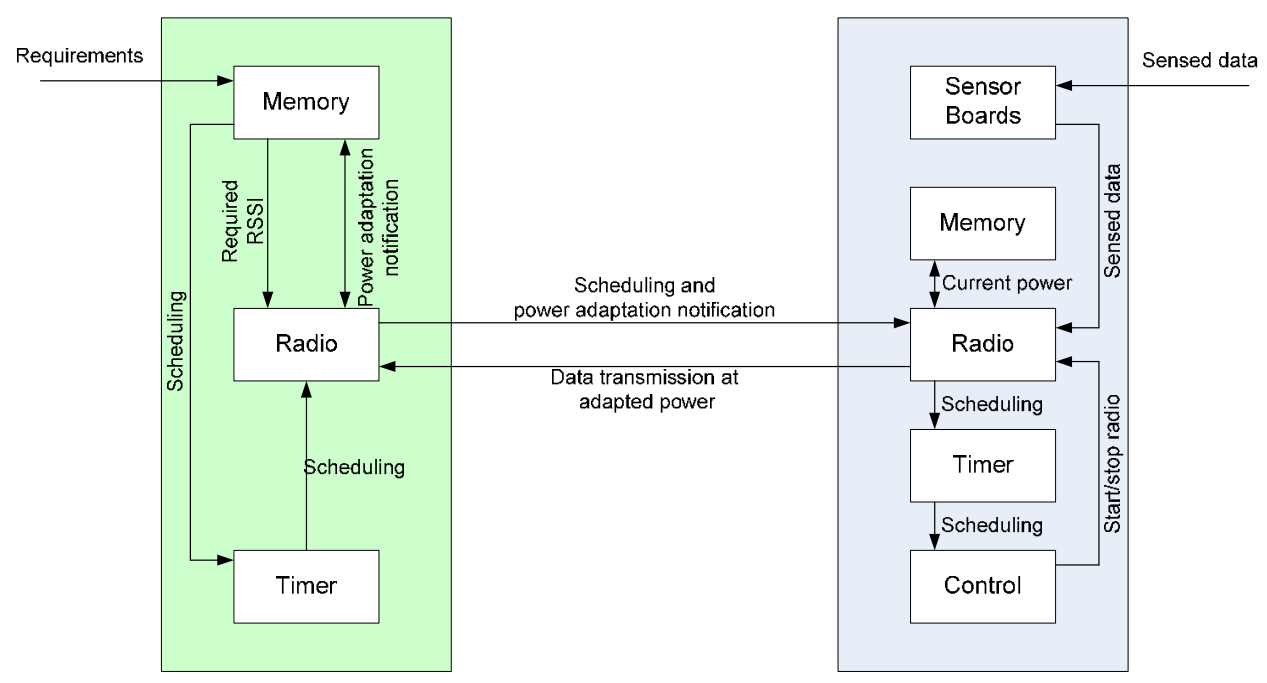

Fig. 12. Interactions between components

\section{Base station}

The base station acts as a destination for the data. The requirements are stored in the memory and they are used to set required RSSI range and the data sending rate. In PoRAP, the schedule-based scheme is adopted where each source has its own slot for data transmission. The slot must be large enough to accommodate several communication delays. According to the results in Section 4.3.2, sending and receiving delays are mainly dependent upon the packet size whereas the two-way propagation delay is significantly small. Models are required for estimating the slot size and they will be described later in this chapter. The next transmission begins after the other sources have already transmitted. Hence, PoRAP suits the applications which require a low duty cycle. The timer is used for scheduling the communications so it also uses this requirement from the application.

The required RSSI range can be obtained from the RSSI-PRR relationship which is dependent upon different conditions such as time-of-day, environment and location of deployment. The PRR is also used as an additional link quality metric as it is close to the reliability requirement. The main objective of PoRAP is to conserve communication energy whilst data loss is minimised. In the short term, the base station measures the RSSI when it receives the data packet. It uses the observed RSSI to determine whether power adaptation is required. The notification bits which are reserved for each source are then set. In the medium or longer term, the base station measures the PRR and uses that to determine what the upper and lower RSSI bounds should be. If more packets are lost, the RSSI bounds are increased. However, the bounds are slowly lowered to reduce power expenditure if the loss is low or non-existence. The number of notification bits is crucial as the base station has to communicate with all the sources in its range. Using too many bits may lead to a control packet which is larger than the buffering capacity of the radio chip.

The base station radio is not started or stopped as it has to continually receive the data packets from its sources. Data packet receptions occur after broadcasting the control packet at the maximum transmission power level. This concept is feasible as the base station has an 
extra source of power from its connecting computer. In PoRAP, the power conservation goal is mainly located at the sources.

\section{Source}

In WSN, the source is responsible for physical data collection. The data is then transmitted to the base station. The key objective of PoRAP is to conserve communication power of the source. Prior to transmission, the source determines whether it has to adapt its current power. The notification is included in the control packet and it is received by the radio of the source. As the buffering capacity of the radio is limited, the base station notifies what the source should do to its current power instead of specifying the appropriate power level. Thus, the source has to store the current power in the memory. For example, the current power is increased if a lower RSSI is measured by the base station. Moreover, the source should recognise the limitations of the transmission power adaptation. The base station may need its source to increase the power even if the maximum has already been reached. The minimum and maximum power levels are dependent upon the selected radio chip.

Apart from the power adaptation signaling, the scheduling is also included in the control packet. Time synchronisation is crucial in the schedule-based approach. The local clock of each node may run at different speeds. In PoRAP, the sources synchronise with their base station. The synchronisation refers to several timestamps which are conducted at the MAC layer where hardware and operating system dependent delays can be disregarded. The scheduling is also recognised by timer and controls components. Several timers are required as they are responsible for timing the sending and receiving communications. The timers operate closely with the control in order to start and stop the radio. For example, the radio is stopped after the data packet is sent. The source knows when it has to wake up to receive the next control packet. The timer is then started, counting the generated ticks. A control interface is used to start the radio for control reception when the scheduled time has come.

\subsubsection{Transmission power adaptation policies}

A sensor consists of hardware components working together to facilitate sensing, processing and communicating tasks. Amongst these components, the transceiver or radio unit is responsible for data communication. Normally, the radio unit supports programmable transmission power and the possible adaptable range is given in the datasheet. For example, the Tmote sensor platform which is chosen for this work employs the CC2420 radio. The minimum and maximum powers are 0 and $-25 \mathrm{dBm}$, respectively. There are two main factors which should be taken into account when transmission power adaptation is required. Several hardware limitations of the radio unit include the allowable minimum, maximum transmission power and base noise. The environmental factors leading to signal strength attenuation should be determined. The selected transmission power should be high enough to produce the associated receiving strength which is not discarded by the receiving node. The maximum power allowed by the radio unit is used as the upper limit. In PoRAP, sources use maximum power for their first transmissions. This policy ensures that the packets will likely be transmitted to the base station. However, both base noise and attenuation are respectively hardware and environment dependent. It is difficult to specify an accurate power adaptation level which can be generally used. Moreover, additional resources will be required if the sources periodically measure and send their base noise to the base station. Attenuation is hard to predict as link quality changes over time. Hence, 
PoRAP repetitively increases or decreases the transmission power within an allowable range instead of discovering the right power.

\subsubsection{Frame structure and slot decomposition}

In PoRAP, a frame is used to represent a communication cycle which consists of one control slot at the beginning followed by several data slots. Its structure is shown in Fig. 13.

$G$ indicates the guard of the frame and is used to protect frame overlapping. A control slot is used by the base station for broadcasting control data which includes scheduling information and transmission power (TX) adaptation notification to its sources. The slot information is required by the sources in order to synchronise themselves to the base station. The time of starting the first data slot is required so that the sources know when data is sent. In PoRAP, each slot has the same length which should accommodate a specific data payload size to be completely transmitted and received.

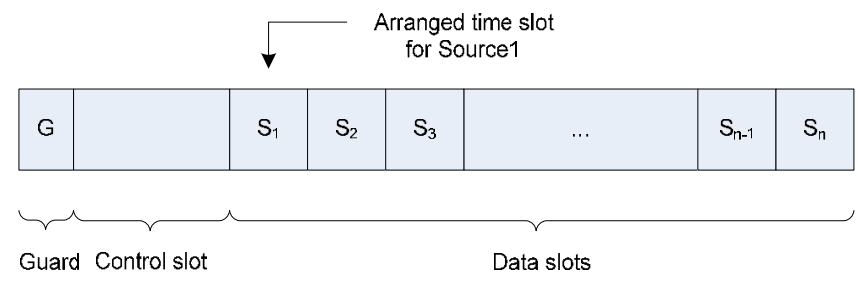

Fig. 13. Frame structure

According to Fig. 13, the sources firstly turn their radios on during the control slot to receive the control information. If they are not assigned to the first data slot, they stop the radios after knowing when their slots start. When their slots arrive, the radios are re-started to send the data. Unlike sources, the base station listens to the medium for data packet reception all the time. The decomposition of a slot is depicted in Fig. 14.

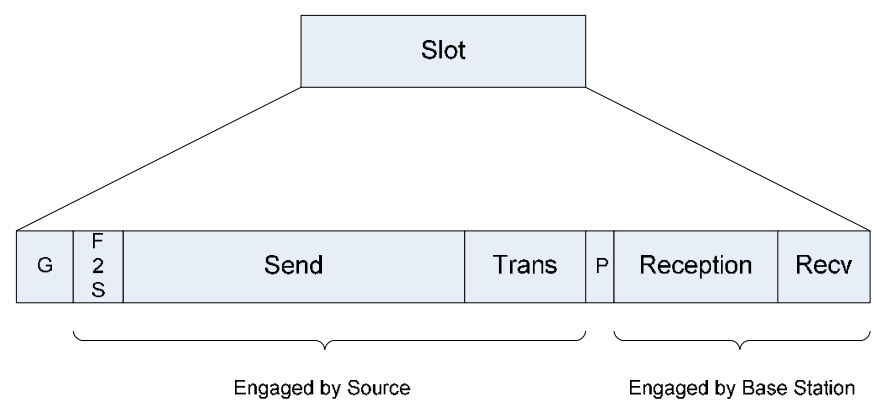

Fig. 14. Data slot decomposition

There are four main delay components in Fig. 14. The $G$ and $P$ are respectively the guard time and propagation delay. The first component is the guard length which prevents the slots from overlapping. Feasible overlapping scenarios together with guard time consideration are provided later in this section. The second component consists of fire-tosend (F2S), send and transmission delays and this is the sending delay component. This 
component is caused by the source. The third one is propagation delay which is considerably smaller than the other delays. Finally, the receiving delay component includes the reception and receive delays. This component is considered during packet arrival at the base station.

\subsubsection{Estimation of communication delays}

A schedule-based approach is adopted in PoRAP. The base station allocates and manages several time slots. In this work, a set of fixed nodes is determined. The number of data slots is therefore equal to the number of booted sources which are able to receive the control packet broadcast by the base station. The source initiates transmission when its assigned slot arrives. Apart from data slots, a frame also contains a control slot which is used by the base station. The slot must be large enough to accommodate sending and receiving delays to avoid feasible data collisions. As shown in Section 4.3.2, the delays are dependent upon packet sizes. This section analyses these relationships for delay estimations.

The experimental results on delays described in Section 4.3.2 demonstrate linear relationships between delays and data packet sizes. The key objective in this part is to discover the two coefficients obtained from linear regression analyses. The coefficients will be used to establish the models providing estimated delays where payload sizes are input. In total 5 payload sizes including 39, 55, 75, 95 and 115 bytes were varied to investigate changes in delays. Regression analyses have been applied to the results of the sending and receiving delays of the source and the base station. As linear relationships between delays and payload sizes are observed, two coefficients of the linear equation $\left(c_{0}\right.$ and $\left.c_{1}\right)$ are the required output where $c_{0}$ is the y-intercept and $c_{1}$ is the slope. The Table 6 summarises the coefficients of each delay.

According to Table 6, the coefficients for the base station do not significantly differ from those for the source. The fire-to-send delays of the base station are constant whilst the source provided a linear relationship.

\begin{tabular}{|l|c|c|c|}
\hline \multirow{2}{*}{ Delays } & \multirow{2}{*}{ Measured at } & \multicolumn{2}{c|}{ Coefficients } \\
\cline { 2 - 4 } & & $\boldsymbol{c}_{\boldsymbol{0}}$ & $\boldsymbol{c}_{\boldsymbol{1}}$ \\
\hline \multirow{2}{*}{ 1. Fire-to-send (F2S) } & Base station & \multicolumn{2}{c|}{ Constant delays of $0.50 \mathrm{~ms}$} \\
\hline \multirow{2}{*}{ 2. Send } & Source & 0.204 & 0.025 \\
\hline \multirow{2}{*}{ 3. Transmission } & Base station & 11.367 & 0.043 \\
\cline { 2 - 4 } & Source & 11.263 & 0.043 \\
\hline \multirow{2}{*}{ 4. Reception } & Base station & 0.490 & 0.033 \\
\cline { 2 - 4 } & Source & 0.552 & 0.033 \\
\hline \multirow{2}{*}{ 5. Receive } & Base station & 1.521 & 0.076 \\
\cline { 2 - 4 } & Source & 1.521 & 0.076 \\
\cline { 2 - 4 } & Base station & \multicolumn{2}{c|}{ Constant delays of $0.22 \mathrm{~ms}$} \\
\hline
\end{tabular}

Table 6. Coefficients obtained from experimental results at $99^{\text {th }}$ percentile

In the case where the payload size is zero, a specific duration is still required for header transmission and reception. For CC2420, the header is approximately 11 bytes and requires $0.352 \mathrm{~ms}$ for the delivery. An additional duration is required for transmitting processes which can be considered as an overhead. The send delay is the largest of the experimental 
results. It is an interval from calling the send() command until capturing the SFD. Several mechanisms undertaken by the application software and operating system to facilitate the sending also require time and are included in the send delay. For example, when the send() command is called by the application, an interrupt is signaled to TinyOS. The packet is buffered and the CC2420 is switched to transmitting mode. This sending overhead due to software manipulation and hardware setup is regardless of payload size. Increases in payload size require additional delays. For example, for every byte increase in the payload size, the send and reception delays of a source respectively increase by 0.043 and $0.076 \mathrm{~ms}$. However, the payload size does not affect receive delay. The coefficients can be used to estimate the communication delays.

\subsubsection{PoRAP scenario}

PoRAP is developed to effectively support data communication in single-hop wireless sensor network (WSN). The base station communicates with its sources for controlling and data collection purposes. As the base station does not know when each source is booted, a setup process is required at the beginning of frame structure. Acting as a data receiver, the base station always listens to the medium for incoming messages after broadcasting the control packet. Hence, the base station desires extra power which can be obtained from external sources such as a desktop or laptop computer.

\section{A) Control and setup phase}

Prior to data transmission, the sources have to setup their parameters based upon the control information received from their base station. The information such as number of slots, slot length and slot start time is used to control the sources in order to send data within an allocated slot at an adapted transmission power. As the base station has no information on when the sources join the network, it has to discover which sources are booted and ready for communication. In the control and setup phase, the base station periodically broadcasts control packets to all sources located in its communication range. The broadcasted packet is received by the booted sources and they use the received information to setup the communication parameters.

There are three main parts to the control information included in the control packet. The first attribute indicates the identification of the base station. This field supports a future enhancement of PoRAP which supports the multiple base station system. It can be also used to differentiate between the control and data packets. The second attribute is schedule related. Some information is required by the sources in order to synchronise with their base station. These parameters include the number of slots, slot length and the start time of the first slot. The base station specifies the slot start time with respect to the Start of Frame Delimiter (SFD) transmission in order to minimise the effects of application and hardware processing delays. The source assigned to the first data slot sets its timer to fire and sends data when the time arrives. Other sources start at different times and they compute the starting times from the slot information. The transmission parameters are required to be completely set before the phase begins. Slot length determination for data slot can therefore be applied to the control slot. The base station periodically broadcasts its control packet. There are two main objectives of periodic broadcasting are maintaining synchronisation between nodes and supporting changes in network topology. Additional sources may be booted during the frame and some sources may be running out of energy. The number of sources is therefore modified by the base station. 


\section{B) Data delivery phase}

Slots are allocated by the base station in order to facilitate data transmissions of the sources. The data delivery phase starts after the control packet is received by the sources. The number of slots is fixed as it assumes that the base station communicates with the fixed number of sources and the number is constant throughout the operation. Data collected by the sources is stored in the data packet and is delivered to the base station.

The Received Signal Strength Indicator (RSSI) is measured when the base station receives the data. The RSSI linearly relates to the transmission power and the RSSI-PRR relationship is established in Fig. 5 (a). The PRR steeply increases with the RSSI up to a certain point. The increase in PRR then becomes insignificant or it becomes constant after this point. The RSSI is monitored and compared to the desired range. Power adaptation notification is conducted by the base station. The sources are notified by control packet reception in the next frame.

Apart from data, the identification (id) of a source is also included in the data packet. Specifying source id is an important issue and it may be done in several ways. For example, the SFD of the control packet reception time may be modified to obtain the id. However, sensors are considered resource constrained. Simple calculations should be included in the sources. Within the 128-byte buffering limitation in CC2420, one to two bytes should be enough to represent the id. Furthermore, the id can be assigned at installation time. Prior to deployment, a particular id is allocated to the source. For example, an id of 1 may be used for installing PoRAP in the first source in the network. Additional power conservation is introduced during the data delivery phase. The strategy benefits from adopting the time-slot based concept. As sources know when to receive control and to transmit data packets, it is possible to periodically turn the radio on for such periods. Fig. 15 describes the mode switching concept during the data delivery phase. The C\&S, R, S and G represent control and setup, receive, send and guard, respectively.

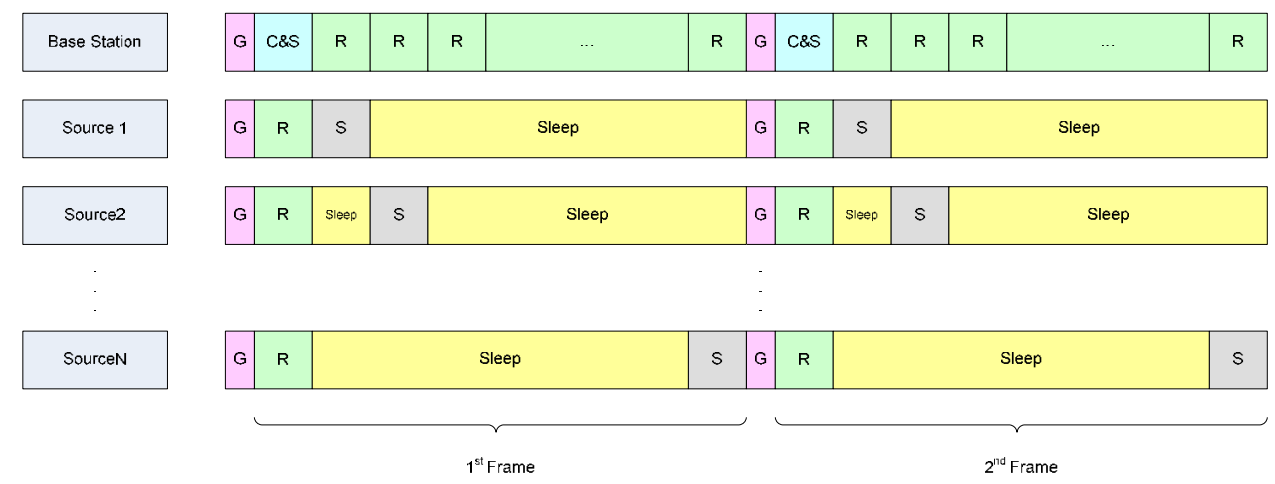

Fig. 15. Mode switching during the data delivery phase

According to Fig. 15, each source is in wakeup mode when its radio is turned on for two reasons; control packet reception and data packet transmission. Otherwise, its radio is turned off and the source is switched to sleep mode. However, the base station radio is always turned on. This strategy minimises idle listening power at the sources. 


\section{PoRAP energy conservation evaluation}

An experiment was conducted in a $16 \mathrm{~m} \times 20 \mathrm{~m}$ indoor environment to evaluate the energy conservation of PoRAP. A network consisting of 20 sources and a base station was set up. Tmote Sky motes were used as both sources and base station. The sources were placed at 20 different locations with 14 different distances and the base station was connected to a desktop machine. All motes had the same height above ground level and had the same antenna orientation. The minimum and maximum distances are 1 and $22.5 \mathrm{~m}$, respectively. Initially, the base station broadcast its 18-byte control packet to the sources. The sources then transmitted the 48-byte data packets back to the base station. A communication cycle was completed after the base station had received the data from all sources. Apart from the maximum power settings, four additional RSSI settings are included. The minimum RSSI thresholds were set to $-90,-80,-70$ and $-60 \mathrm{dBm}$ whereas the corresponding maximum thresholds were $-80,-70,-60$ and $-50 \mathrm{dBm}$, respectively. The power is not adapted if the measured RSSI is between the thresholds and the aim is to obtain nearly 100\% PRR. Each mote transmitted every 5 minutes and the experiment lasted for 24 hours. The results are shown in Table 7.

\begin{tabular}{|c|c|c|c|c|c|c|c|c|c|c|}
\hline \multirow[b]{2}{*}{$\begin{array}{l}\text { Dist. } \\
\text { (m) }\end{array}$} & \multicolumn{2}{|c|}{$-90<$ RSSI $<-80$} & \multicolumn{2}{|c|}{$-80<\mathrm{RSSI}<-70$} & \multicolumn{2}{|c|}{$-70<$ RSSI $<-60$} & \multicolumn{2}{|c|}{$-60<$ RSSI $<-50$} & \multicolumn{2}{|c|}{$\operatorname{Max} T X$} \\
\hline & $\begin{array}{c}\text { Saved } \\
\text { Trans } \\
\text { Current }\end{array}$ & $\begin{array}{c}\text { Packet } \\
\text { Loss } \\
(\%) \\
\end{array}$ & $\begin{array}{c}\text { Saved } \\
\text { Trans } \\
\text { Current }\end{array}$ & $\begin{array}{c}\text { Packet } \\
\text { Loss } \\
(\%) \\
\end{array}$ & $\begin{array}{c}\text { Saved } \\
\text { Trans } \\
\text { Current }\end{array}$ & $\begin{array}{c}\text { Packet } \\
\text { Loss } \\
(\%) \\
\end{array}$ & $\begin{array}{c}\text { Saved } \\
\text { Trans } \\
\text { Current }\end{array}$ & $\begin{array}{c}\text { Packet } \\
\text { Loss } \\
(\%)\end{array}$ & $\begin{array}{c}\text { Saved } \\
\text { Trans } \\
\text { Current }\end{array}$ & $\begin{array}{c}\text { Packet } \\
\text { Loss } \\
(\%) \\
\end{array}$ \\
\hline 1 & 51.2 & 0 & 51.2 & 0 & 51.2 & 0 & 35.6 & 0 & 0 & 0 \\
\hline 2 & 51.2 & 0.3 & 35.6 & 0.7 & 0 & 0 & 0 & 0 & 0 & 0 \\
\hline 4 & 43.1 & 2.3 & 43.1 & 0.7 & 28.2 & 0 & 0 & 0 & 0 & 0 \\
\hline 6 & 43.1 & 4.7 & 28.2 & 0 & 0 & 0.3 & 0 & 0 & 0 & 0 \\
\hline 8 & 51.2 & 5 & 0 & 0.7 & 0 & 0.3 & 0 & 0 & 0 & 0 \\
\hline 10 & 51.2 & 5.3 & 35.6 & 0 & 0 & 0 & 0 & 0 & 0 & 0 \\
\hline 12 & 51.2 & 5.7 & 20.1 & 0 & 0 & 0 & 0 & 0 & 0 & 0 \\
\hline 14 & 0 & 14 & 28.2 & 0 & 0 & 0 & 0 & 0.4 & 0 & 3.7 \\
\hline 16 & 28.2 & 5.7 & 20.1 & 0 & 0 & 0 & 0 & 0 & 0 & 1.2 \\
\hline 20 & 43.1 & 3.7 & 0 & 0.7 & 0 & 0 & 0 & 1.2 & 0 & 2.1 \\
\hline
\end{tabular}

Table 7. Conserved transmitting current and data packet loss

According to Table 7, lower RSSI settings result in higher percentage of packet loss and conserved transmitting power. Lower power is used to produce the required RSSI range. A significant amount of power up to $50 \%$ can be yielded. However, the highest packet loss is obtained when the RSSI is between -90 and $-80 \mathrm{dBm}$.

\section{Conclusion}

This chapter describes several aspects which should be considered during developing a network protocol for wireless sensor network (WSN). WSN has been used in both surveillance and civil applications. It is considered application specific as each application has its own set of requirements. Two main categories are proposed including event-based and periodic-based application. Throughput is the key requirement in the event-based whilst lifetime is the key in the periodic-based. Moreover, one of major drawbacks of WSN 
is resource constraint. The power for all operations comes from tiny batteries. Under some circumstances, it is uneconomical or impractical to change or recharge the batteries. In WSN, the data is delivered via wireless link which is susceptible to the surrounding environments. The radio unit is responsible for data delivery has a limited buffering capacity. Control information should be minimised to be included in a packet.

The Power \& Reliability Aware Protocol (PoRAP) is developed and its main objective is to provide an efficient data communication by means of energy conservation whilst reliability is maintained. Its three key elements include direct communication, adaptive transmission power and intelligent scheduling. With adaptive transmission power and intelligent scheduling, the power consumption is minimised as a result of a lower transmitting power, collision avoidance and minimised idle listening without unnecessary data losses. The key capabilities of PoRAP make it suitable for use in the periodic-based WSN applications with regular reporting patterns where maximising bandwidth is not the prime concern. PoRAP thus applies to some of the WSN applications such as environmental and habitat monitoring where the sources often remain at their positions throughout the operation. Slots are allocated to the sources for data transmissions. In PoRAP, it is assumed that the number of allocated slots is equal to that of sources. A low duty cycle application is more efficient using PoRAP when the percentage of slot usage is high. The evaluation results indicate up to $50 \%$ of power can be yielded whilst the reliability is within the desired range. However, PoRAP is not applicable if a source has to wait longer until the next cycle is started. Therefore, a limitation of PoRAP arises when there is a high slot overhead because there are many sources in the network.

\section{References}

Warneke, B. \& Pister, K.S.J. (2002). MEMS for Distributed Wireless Sensor Networks, Proceeding of the 9th International Conference on Electronics, Circuits and Systems. Dubrovnik, Croatia

Mainwaring, A.; Polasrte, J. ; Szewczyk, R.; Culler, D. \& Anderson, J. (2002). Wireless Sensor Networks for Habitat Monitoring, WSNA'02, Atlanta, Georgia, USA.

Allen, G.W.; Lorincz, K.; Ruiz, A.; Marcillo, O.; Johnson, J.; Lees, J. \& Welsh, M. (2006). Deploying a Wireless Sensor Network on an Active Volcano. IEEE Internet Computing, Vol.10, No.2, pp.18-25

Essa, I.A. (2000). Ubiquitous Sensing for Smart and Aware Environments. IEEE Personal Communications

Srivastava, M.; Muntz, R. \& Potkonjak, M. (2001). Smart Kindergarten: Sensor-Based Wireless Networks for Smart Developmental Problem-Solving Environments. ACM SIGMOBILE, Rome, Italy

Jovanov, E.; O'Donnell Lords, A.; Raskovic, D.; Cox, P.G.; Adhami, R. \& Andrasik, F. (2003). Stress Monitoring Using a Distributed Wireless Intelligent Sensor System. IEEE Engineering in Medicine and Biology Medicine

Otto, C.; Milenković, A.; Sanders, C. \& Jovanov, E. (2006). System Architecture of a Wireless Body Area Sensor Network for Ubiquitous Health Monitoring, Journal of Mobile Multimedia, Vol.1, No.4, pp.307-326

Arora, A.; Dutta, P.; Bupat, S.; Kulathumani, V.; Zhang, H.; Naik, V.; Mittal, V.; Cao, H.; Demirbas, M.; Gouda, M.; Choi, Y.; Herman, T.; Kulkarni, S.; Arumugam, U.; Nesterenko, M.; Vora, A. \& Miyashita, M. (2004). A Line in the Sand: A Wireless 
Sensor Network for Target Detection, Classification, and Tracking. Computer Networks: The International Journal of Computer and Telecommunications Networking, Vol.46, Issue 5, pp.605-634

Chintalapudi, K.; Fu, T.; Paek, J.; Kothari, N.; Rangwala, S.; Caffrey, J.; Govindan, R.; Johnson, E. \& Masri, S. (2006). Monitoring Civil Structures with a Wireless Sensor Network, IEEE Internet Computing

Juang, P.; Oki, H.; Wang, Y.; Martonosi, M.; Peh, L.S.; \& Rubenstein, D. (2002). EnergyEfficient Computing for Wild-Life Tracking: Design Tradeoffs and Early Experiences with ZebraNet, ASPLOS'02, ACM

Szewczyk, R.; Mainwaring, A.; Polasrte, J.; Anderson, J. \& Culler, D. (2004). An Analysis of a Large Scale Habitat Monitoring Application, SenSys'04, Baltimore, Maryland, USA

Martinez, K.; Padhy, P.; Riddoch, A.; Ong, H.L.R. \& Hart, J.K. (2005). Glacial Environment Monitoring Using Sensor Networks, REALWSN'05, Stockholm, Sweden

Kottapalli, V.A.; Kiremidjian, A.S.; Lynch, J.P.; Carryer, E.; Kenny, T.W.; Law, K.H. \& Lei, Y. (2003). Two-Tiered Wireless Sensor Network Architecture for Structural Health Monitoring, Proceedings of SPIE's 10 th Annual Symposium on Smart Structures and Materials, San Diego, USA

Paek, J.; Chintalapudi, K.; Caffrey, J.; Govindan, R. \& Masri, S. (2005). A Wireless Sensor Network for Structural Health Monitoring: Performance and Experience, Proceedings of the $2^{\text {nd }}$ IEEE Workshop on Embedded Networked Sensors (EmNetS-II), Sydney, Australia

Schmid, T.; Dubois-Ferrière, H. \& Vetterli, M. (2005). SensorScope: Experiences with a Wireless Building Monitoring Sensor Network, REALWSN'05, Stockholm, Sweden

Dreicer, J.S.; Jorgensen, A.M. \& Dors, E.E. (2002). Distributed Sensor Network with Collective Computation for Situational Awareness, AIP Conference Proceedings, Vol.632, pp.235-243

Simon, G.; Balogh, G.; Pap, G.; Maróti, M.; Kusy, B.; Sallai, J. ; Lédeczi, Á.; Nádas, A. \& Frampton, K. (2004). Sensor Network-Based Countersniper System, SenSys'04, Maryland, USA

Coleri, S.; Cheung, S.Y. \& Varaiya, P. (2004). Sensor Networks for Monitoring Traffic, University of California Berkeley Technical Report, August 2004

Brignone, C.; Conners, T.; Lyon, G. \& Pradhan, S. (2005). SmartLOCUS: An Autonomous, Self-Assembly Sensor Network for Indoor Asset and Systems Management, Hewlett-Packard Development Company Technical Report, June 2005

Sankarasubramaniam, Y.; Akan, O.B. \& Akyildiz, I.F. (2003). ESRT: Event-to-Sink Reliable Transport in Wireless Networks, ACM MobiHoc'03, Maryland, USA

Ee, C.T. \& Bajcsy, R. (2004). Congestion Control and Fairness for May-to-One Routing in Sensor Networks, ACM SenSys'04, Bultimore, Maryland, USA

Hull, B.; Jamieson, K. \& Balakrishnan, H. (2004). Mitigating Congestion in Wireless Sensor Networks, ACM SenSys'04, Bultimore, Maryland, USA

Lu, C.; Blum, B.M.; Abdelzaher, T.F.; Stankovic, J.A. \& He, T. (2002). RAP: A Real-Time Communication Architecture for Large-Scale Wireless Sensor Networks, RTAS, September 2002

Wan, Chieh-Yih; Eisenman, S.B. \& Campbell, A.T. (2003). CODA: Congestion Detection and Avoidance in Sensor Networks, ACM SenSys'03, Los Angeles, USA 
Wan, Chieh-Yih; Campbell, A.T. \& Krishnamurthy, L. (2002). PSFQ: A Reliable Transport Protocol for Wireless Sensor Networks, ACM WSNA'02, Atlanta, Georgia, USA

Stann, F. \& Heidemann, J. (2003). RMST: Reliable Data Transport in Sensor Networks, IEEE International Workshop on Sensor Net Protocols and Applications (SNPA), Anchorage, USA

Intanagonwiwat, C.; Govindan, R.; Estrin, D. \& Heidemann, J. (2003). Directed Diffusion for Wireless Sensor Networking, IEEE/ACM Transactions on Networking, Vol.11, No.1, February 2003

Xu, N.; Rangwala, S.; Chintalapudi, K.K.; Ganesan, D.; Broad, A.; Govindan, R. \& Estrin, D. (2004). A Wireless Sensor Network for Structural Monitoring, ACM SenSys'04, Baltimore, Maryland, USA

Polastre, J.; Hill, J. \& Culler, D. (2004). Versatile Low Power Media Access for Wireless Sensor Networks, SenSys'04, November 2004

Tolle, G.; Polastre, J.; Szewczyk, R.; Culler, D.; Turner, N.; Tu, K.; Burgess, S.; Dawson, T.; Buonadonna, P.; Gay, D. \& Hong, W. (2005). A Macroscope in the Redwoods, SenSys'05, November 2005

Shnayder, V.; Hempstead, M.; Chen, Bor-rong; Allen G.W.; \& Welsh, M. (2004). Simulating the Power Consumption of Large-Scale Sensor Network Applications, SenSys'04, Baltimore, Maryland, USA

Lin, S.; Zhang, J.; Zhou, G.; Gu, L.; He, T. \& Stankovic, J.A. (2006). ATPC: Adaptive Transmission Power Control for Wireless Sensor Networks, SenSys'06, Boulder, Colorado, USA

Stoyanova, T.; Kerasiotis, F.; Prayati, A. \& Papadopoulos, G. (2007). Evaluation of Impact Factors on RSS Accuracy for Localization and Tracking Applications, In MobiWac'07, October 2007

Srinivasan, K.; Dutta, P.; Tavakoli, A. \& Levis, P. (2006). Understanding the Causes of Packet Delivery Success and Failure in Dense Wireless Sensor Networks, Technical Report SING-06-00, Stanford University 


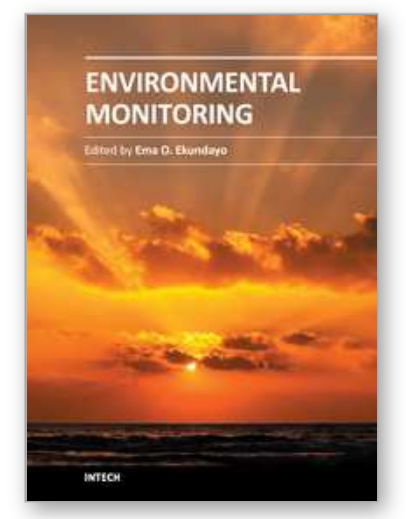

\author{
Environmental Monitoring \\ Edited by Dr Ema Ekundayo
}

ISBN 978-953-307-724-6

Hard cover, 528 pages

Publisher InTech

Published online 04, November, 2011

Published in print edition November, 2011

"Environmental Monitoring" is a book designed by InTech - Open Access Publisher in collaboration with scientists and researchers from all over the world. The book is designed to present recent research advances and developments in the field of environmental monitoring to a global audience of scientists, researchers, environmental educators, administrators, managers, technicians, students, environmental enthusiasts and the general public. The book consists of a series of sections and chapters addressing topics like the monitoring of heavy metal contaminants in varied environments, biolgical monitoring/ecotoxicological studies; and the use of wireless sensor networks/Geosensor webs in environmental monitoring.

\title{
How to reference
}

In order to correctly reference this scholarly work, feel free to copy and paste the following:

Ittipong Khemapech (2011). Environmental Monitoring WSN, Environmental Monitoring, Dr Ema Ekundayo (Ed.), ISBN: 978-953-307-724-6, InTech, Available from: http://www.intechopen.com/books/environmentalmonitoring/environmental-monitoring-wsn

\section{INTECH}

open science | open minds

\section{InTech Europe}

University Campus STeP Ri

Slavka Krautzeka 83/A

51000 Rijeka, Croatia

Phone: +385 (51) 770447

Fax: +385 (51) 686166

www.intechopen.com

\section{InTech China}

Unit 405, Office Block, Hotel Equatorial Shanghai

No.65, Yan An Road (West), Shanghai, 200040, China

中国上海市延安西路 65 号上海国际贵都大饭店办公楼 405 单元

Phone: +86-21-62489820

Fax: +86-21-62489821 
(C) 2011 The Author(s). Licensee IntechOpen. This is an open access article distributed under the terms of the Creative Commons Attribution 3.0 License, which permits unrestricted use, distribution, and reproduction in any medium, provided the original work is properly cited. 